\title{
Development of a new nanofiltration membrane for removal of kinetic hydrate inhibitor from water
}

\author{
Maryam Golpour, Majid Pakizeh* \\ Department of Chemical Engineering, Faculty of Engineering, Ferdowsi University of Mashhad, P.O. Box \\ 9177948974, Mashhad, Iran
}

\begin{abstract}
Kinetic Hydrate Inhibitors (KHIs) are added to the produced fluids in natural gas fields to prevent hydrate formation. Because KHI dissolved into produced water may have a negative impact on the environment, an effective treatment method is needed to remove KHI. A novel thin film nanocomposite (TFN) nanofiltration (NF) membrane was prepared with polyphenylsulfone (PPSU)/graphene oxide (GO) blended support membrane fabricated by the phase inversion method and with a polyamide (PA) selective layer formed by interfacial polymerization over the surface of the PPSU (TFC) and PPPSU/GO supports. The performance of the NF membranes was investigated in terms of the pure water flux (PWF), $\mathrm{MgSO}_{4}$ rejection and KHI rejection under different operating pressures and feed concentrations. The TFN membrane embedded with 0.1 wt. \% GO exhibited higher permeate flux than the TFC membrane, with no significant change in KHI rejection. The KHI rejection of $99 \%$ and permeation flux of $32.7 \mathrm{~L} / \mathrm{m}^{2}$.h (at 9 bar and feed concentration of 0.5 wt. \% KHI) were achieved, suggesting that the TFN membrane has potential as an application for KHI removal from water. The prepared TFN membrane also showed a significant antifouling property due to the improved smoothness of the membrane surface.
\end{abstract}

Keywords: Kinetic hydrate inhibitor (KHI), Nanofiltration (NF), Thin film nanocomposite (TFN), Graphene oxide (GO), Polyphenylsulfone (PPSU)

\footnotetext{
* Corresponding author. Tel./fax: +98 5118816840.

E-mail address: pakizeh@um.ac.ir (M. Pakizeh).
}

(C) 2016. This manuscript version is made available under the Elsevier user license http://www.elsevier.com/open-access/userlicense/1.0/ 


\section{Introduction}

During the extraction of hydrocarbons (oil and/or natural gas), large amounts of water are produced concurrently with hydrocarbons, called "produced water". This water contains various organic and inorganic contaminant compounds [1]. These chemicals, individually or collectively, could have significant impact on the environment, so the produced water has to be further treated before it can be discharged. One of these chemicals is kinetic hydrate inhibitors (KHIs) that are added to hydrocarbon production flow to prevent hydrate formation during extraction.

Natural gas hydrate is an ice-like solid that can form during the transportation of natural gas in the existence of water under certain thermodynamic conditions (usually high pressure and low temperature). Hydrates can plug the piping, pieces of hydrate moving through equipment or pipes. To counter this problem, KHIs have been in commercial use in the upstream oil and gas industry since 1995 [2,3]. These inhibitors are water-soluble polymers, sometimes with additional organic molecules that delay the nucleation and usually the crystal growth of gas hydrates [4]. Unfortunately, KHIs used during hydrocarbon production often contaminate the produced water. Due to environmental concerns and/or regulations, it is best to remove these inhibitors before discharging the contaminated water. Some of the conventional separation methods include solvent extraction, adsorption, electro-coagulation, chemical coagulation, and advanced oxidation processes; however, many of the conventional methods are limited to removing only a low percentage of inhibitors from water (e.g. solvent extraction, and chemical coagulation) [5]. In some cases, these methods suffer from complexity, intensive capacity limitations, excessive high capital, and/or excessively high operational costs.

Talley and Colle [6] described a separation method for removing hydrate inhibitors from an aqueous phase, which involves heating the aqueous phase to a temperature above the boiling point of the water. From an energy consumption perspective, this method is not efficient and can lead to problems in removing a polymer with cloud point below the boiling temperature. Adham and his group [7-9] used various physical, biological, and chemical treatment methods for removing KHI from synthetically produced water, including heated centrifugation, biotreatment, advanced oxidation process, and membrane filtration. The centrifugation of $1.5 \% \mathrm{KHI}$ solution at $65{ }^{\circ} \mathrm{C}$ removed $45 \%$ of the $\mathrm{KHI}$, and the biotreatment was capable of removing $50-60 \%$ of the chemical oxygen demand (COD) associated with KHI [7]. The oxidation with ozone or Fenton's reagent can remove up to $95 \%$ of the KHI from a $0.25 \%$ KHI solution [8]. The ultrafiltration 
(UF), nanofiltration (NF) and reverse osmosis (RO) commercial membranes used to reject the KHI from a synthesis produced water and according to the results RO and NF membranes were successful in separation of the KHI with a removal rate of about 99\% [7,9]. In other studies, ceramic membranes were tested for KHI removal [5,10]. Blumer et al [5] used the composite inorganic membrane comprised of a ceramic, such as alumina, zirconia, silica, silicon carbide, and mixed oxides having molecular weight cut-off or pore sizes from about 1,000 Daltons to about 10 microns for filtering KHI. The inorganic membranes achieved a KHI removal rate greater than approximately 50\% [5,10]. Schrader [11] employed a spirally wound membrane system for separating KHI polymers from an aqueous mixture comprised of hydrocarbons, KHI, and salts. According to the results, the rejection of the KHI was at least $80 \%$ [11].

NF process is rapidly developing for different applications, especially in water and wastewater treatment, because of its advantages such as high flux, low operating pressure, and low operating costs [12]. The most successful NF membranes are thin film composite (TFC) polyamide (PA) membranes with an asymmetric microporous membrane as a support layer and a thin PA layer as a selective layer. The support membrane is generally made by the phase inversion method and the thin film is prepared by the interfacial polymerization (IP) technique invented by Cadotte [13]. The advantage of TFC membrane is that every layer of membrane (i.e. selective layer and/or porous support) can be separately optimized to achieve the best overall separation performance and membrane stability.

In recent years, several studies have been published on the role of support membrane properties in the formation of PA composite membranes [14-22]. In addition to the support membrane provides a mechanical layer for the TFC membrane, the surface hydrophilicity, porosity, and pore size of the support influences the thickness, roughness and cross-linking degree of the PA layer $[14,19,22]$. Recently the incorporation of nanomaterials into the support layer of TFC membrane has attracted considerable attention [23-28]. The physical and structural modifications with these nanomaterials on the support layer caused improved the surface hydrophilicity and/or pore structure, which increased the pure water flux (PWF) of the TFC membranes.

Graphene oxide (GO) is an attractive nanomaterial as a filler for polymer nanocomposites due to its two-dimensional layered structure, oxygenic functional groups, and high surface area [28-40]. The oxygenic functional groups (e.g. carboxyl, carbonyl, hydroxyl, and epoxy groups) in GO offer high potential for making nanocomposite with superior hydrophilicity, antifouling 
properties, and strong chemical stability compared to the neat polymer [28,31-37]. These improved properties encouraged the employment of GO as nanomaterials in nanocomposite membranes. Zhao et al [36] reported that GO blending into polyvinylidene fluoride (PVDF) polymer matrix could improve hydrophilicity, PWF, and antifouling property of the PVDF membrane. Zinadini et al [33] found that the addition of GO to the polyethersulfone (PES) membrane improved the porosity, surface hydrophilicity, PWF, antifouling properties, and solute rejection capability of the membrane. The effects of GO nanosheets incorporation into the polysulfone (PSf) on porosity and pore size of support membrane has been investigated by Jun Park et al [28]. The optimum amount of GO in the PSf support membrane not only increased water permeability of the PSf but also allowed effective thin layer formation. The sulfone polymers (i.e. PSf and PES) are widely used as support due to their superior chemical and mechanical stability [15] Among all sulfone polymers, polyphenylsulfone (PPSU) has better chemical resistance and impact resistance than PSf and PES [41]. Additionally, the high heat resistance and long-term thermal stability of PPSU, introduce it as a remarkable candidate for synthesis of support membranes [42,43].

In this study, GO nanoplates as nanofillers were blended with PPSU as support for membrane samples. To the authors' best knowledge, this is the first report on thin film nanocomposite (TFN) membrane using PPSU/GO nanocomposite substrate for NF applications. One of the objectives of this study is to investigate the effect of the loading of GO nanoplates on the structural characteristics and separation properties of prepared TFN membranes. Another objective is to estimate the ability of this new TFN membrane for separation of $\mathrm{KHI}$ and $\mathrm{MgSO}_{4}$ from water. The characteristics of the prepared TFC and TFN membranes were analyzed by attenuated total reflectance Fourier transform infrared spectroscopy (ATR-FTIR), field emission scanning electron microscopy (FESEM), and atomic force microscopy (AFM). Fouling resistance of the obtained membranes was also studied.

\section{Experimental}

\subsection{Materials}

Polyphenylsulfone (PPSU, Radel R5000) was supplied by SOLVAY (Belgium). A commercial form of multilayer graphene oxide (GO) nanoplates was obtained from Iranian Nanomaterials 
Pioneers Company (Iran). The physicochemical characteristics and microscopic image of the GO nanoplates are shown in Table 1 and Fig. 1, respectively. Piperazine (PIP) and trimesoyl chloride (TMC) were used as aqueous and organic monomers, respectively and obtained from Merck (Germany). N-methyl pyrrolidone (NMP) and n-hexane were also purchased from Merck. $\mathrm{MgSO} 4$ salt supplied by Merck was used to prepare the feed salt solution for NF experiments. Kinetic hydrate inhibitor (KHI) was obtained from NOVINCHEM Co. (Iran) identified LDHI10068. Deionized (DI) water was used throughout this study.

\subsection{Fabrication of TFN-NF membrane}

\subsubsection{Preparation of PPSU/GO support layer}

The nanocomposite support membranes were fabricated by the phase inversion method [44]. The PPSU pellets were dissolved in $35 \%$ of NMP solvent at $60{ }^{\circ} \mathrm{C}$ for $24 \mathrm{~h}$ to form a homogenous polymer solution. To this viscous polymer solution, the desired amount of GO dispersed in the remaining $75 \%$ of NMP by sonication was added to attain various GO concentrations $(0.05,0.1$, 0.2 , and $0.4 \mathrm{wt}$. \%). Next the solution was stirred for $18 \mathrm{~h}$ at $60{ }^{\circ} \mathrm{C}$ and sonicated for $30 \mathrm{~min}$ to ensure homogeneous spread of GO. Table 2 shows the composition for each solution. The casting solution was kept at room temperature without stirring until no bubbles appeared, then the bubble-free solution was casted on (I) a glass plate for characterization and (II) non-woven polyester for PWF and separation experiments using an adjustable casting bar (Neurtek2281205). The film was immediately immersed in a bath of DI water at room temperature. After coagulation, all PPSU/GO membranes were washed thoroughly with water to remove residual solvent and kept wet at $5{ }^{\circ} \mathrm{C}$ prior to use.

\subsubsection{Synthesis of polyamide selective layer}

The polyamide selective layer of NF membranes was synthesized by IP between PIP and TMC on the surface of the PPSU or PPSU/GO as shown in Fig. 2. The substrate was initially immersed in the aqueous solution containing 2\% PIP for 2 min and excessive PIP solution on the substrate surface was removed by a rubber roller. Subsequently, the amine-saturated substrate was immersed in a $0.07 \%(\mathrm{w} / \mathrm{v}) \mathrm{TMC}$ in $\mathrm{n}$-hexane solution for $30 \mathrm{~min}$ to perform the IP process over the substrate. Finally, the resultant membrane was cured at $70{ }^{\circ} \mathrm{C}$ for $10 \mathrm{~min}$ in an oven, and then stored in DI water at $5{ }^{\circ} \mathrm{C}$ before use. These prepared NF membranes were denoted as TFC, 
$\operatorname{TFN}(0.05), \operatorname{TFN}(0.1), \operatorname{TFN}(0.2)$, and $\operatorname{TFN}(0.4)$, respectively. The number corresponds to the loading of GO in the substrate.

\subsection{Membrane characterization}

2.3.1. Fourier transform infrared (FTIR) and attenuated total reflection infrared (ATR-IR) FTIR spectroscope (Thermo Nicolet Avatar 370) was used to characterize the presence of GO in the support membranes. FTIR spectra were collected at wavenumber between $700 \mathrm{~cm}^{-1}$ to 4000 $\mathrm{cm}^{-1}$ with spectral resolution of $1 \mathrm{~cm}^{-1}$. ATR-IR (Thermo Nicolet Avatar 370) was also used to identify the functional groups at the PA selective layer.

\subsubsection{X-ray diffraction (XRD)}

To study the crystalline structure and distribution of the GO on the support membranes, XRD analysis was conducted using a Panalytical X'Pert Pro MPD instrument employing $\mathrm{Cu} \mathrm{Ka}$ radiation at $2 \theta$ ranging from $4^{\circ}$ to $30^{\circ}$.

\subsubsection{Scanning electron microscopy (SEM) and field emission SEM (FESEM)}

The morphology and structure of the support membranes were characterized by SEM (VP 1450, LEO, Germany) at $20 \mathrm{kV}$. The membranes were first cut into small pieces, and then the sample pieces were immersed into liquid nitrogen and broken to obtain a clean cut for cross-sectional view. The sample pieces were then gold sputtered for producing electrical conductivity. A FESEM (TESCAN) was used to study the cross-section and surface morphologies of TFC and TFN membranes.

\subsubsection{Atomic force microscope (AFM)}

The surface roughness of the prepared membranes was evaluated by AFM (full plus series 0101/A, Ara, Iran). Small squares of the prepared membranes were glued on a glass substrate and the membrane surfaces were viewed and analyzed in a scan size of $10 \mu \mathrm{m} \times 10 \mu \mathrm{m}$. Differences on the membrane surface morphology may be expressed in terms of the average roughness $\left(R_{a}\right)$ and the root mean square of the $Z$ data $\left(R_{q}\right)$. 


\subsubsection{Contact angle measurement}

The surface hydrophilic behavior of the PPSU support membranes was characterized through water contact angle measurement. The contact angle between water and surface of membranes was measured using a contact angle goniometer (OCA15plus, Dataphysics) at room temperature. To minimize experimental errors, four measurements were taken at different sites of each membrane sample and the average value was reported.

\subsubsection{Membrane porosity and pore size measurement}

The overall porosity $(\varepsilon)$ of the support membranes was measured using a gravimetric method, as defined in the following equation:

$\varepsilon=\omega 1-\omega 2 A \times l \times \rho w$

Where $\omega 1$ and $\omega 2$ are the wet membrane weight and dried membrane weight, respectively, $A$ $\left(\mathrm{m}^{2}\right)$ is the surface area of the membrane, $l(\mathrm{~m})$ is the membrane thickness and $\rho w$ is the water density $\left(998 \mathrm{~kg} / \mathrm{m}^{3}\right)$.

Using the porosity data and Guerout-Elford-Ferry equation (eqn (2)), the membrane mean pore radius was calculated:

$r m=2.9-1.75 \varepsilon 8 \eta l Q A \times \varepsilon \times \Delta P$

\section{(2)}

where $\eta$ is the water viscosity $(8.9 \times 10-4 \mathrm{~Pa} . \mathrm{s}), Q$ is the volume of permeated pure water per unit time $\left(\mathrm{m}^{3} / \mathrm{s}\right)$, and $\Delta P$ is the transmembrane pressure (bar).

\subsection{Membrane performance evaluation}

A cross-flow filtration system as shown in Fig. 3 was employed for the performance evaluation of the nanocomposite support and TFN-NF membranes. The effective membrane area was around $20 \mathrm{~cm}^{2}$. PWF of the support membranes was measured at 3 bar and $25 \pm 1{ }^{\circ} \mathrm{C}$. The membranes were pressurized with water at 4 bar for $2 \mathrm{~h}$, prior to flux measurements. The flux was calculated by the following equation: 
$J=V A . t$

(3)

where $V(\mathrm{~L})$ is the permeate volume, $A\left(\mathrm{~m}^{2}\right)$ is the effective membrane surface area and $t(\mathrm{~h})$ is the permeate collection time.

The flux and solute rejection of NF membranes were first evaluated using $\mathrm{MgSO}_{4}(2000 \mathrm{ppm})$ feed solution at 8 bar and $25 \pm 1{ }^{\circ} \mathrm{C}$. The membranes were pre-filtrated with water at 8 bar for $1 \mathrm{~h}$ to reach a steady state before testing. The rejection (R) was calculated using eqn (4):

$R(\%)=1-C p C f \times 100$

where $C_{p}$ and $C_{f}$ are the permeate and the feed concentrations (ppm), respectively. The salt concentrations at the feed and permeate solutions were obtained using a conductivity meter (ExStik EC 400, USA).

The KHI separation experiments with TFC and TFN membranes were conducted at three different transmembrane pressures of 5, 7, and 9 bar and three feed concentrations of $0.1,0.5$, and 1 wt. \%. The permeate flux and KHI rejection were calculated according to eqns (3) and (4), respectively. The KHI concentrations at the feed and permeate solutions were measured with a UV-Vis spectrophotometer (Optizen POP, Mecasys, South Korea) at a wavelength of $209 \mathrm{~nm}$. All the prepared membranes (UF and NF) were evaluated at least 3 times in different batches.

\subsection{Antifouling properties of the NF membranes}

The antifouling performance of TFC and TFN membranes was studied with the aqueous solution of 0.5 wt. $\% \mathrm{KHI}$ at 8 bar and $25 \pm 1{ }^{\circ} \mathrm{C}$ in a flat-sheet cross-flow system, as shown in Fig.3. The membrane fouling was calculated by comparing the PWF before and after the KHI solution filtration. The test process was carried out as follows: First, the PWF was measured, and the flux at steady state was defined as $\mathrm{J}_{0}$. Next, the KHI aqueous solution was filtrated through the membrane, and the permeate flux at the end of the experiment was defined as $\mathrm{J}_{1}$. After filtration of the KHI solution, the fouled membrane was washed with water for $10 \mathrm{~min}$. Then, the pure water permeate flux with this washed membrane was measured until a stable permeate flux occurred, and it was defined as $\mathrm{J}_{2}$. The different types of fouling were calculated based on 
equations shown in Table 3 to investigate the fouling process in details [45]. The flux recovery ratio (FRR) was calculated by:

$F R R(\%)=J 2 J 0 \times 100$

\section{Results and discussion}

3.1. Characterization and evaluation of the PPSU/GO support membranes

3.1.1. GO presence in the support membrane

Fig. 4 illustrates the FTIR spectra of GO, the PPSU membrane and the PPSU/GO membranes with 0.1 wt. $\%$ and 0.4 wt. $\%$ GO. The presence of different type of oxygen functionalities in hydrophilic GO was confirmed in Fig. 4a. The spectrum of GO displays a stretching vibration of $\mathrm{O}-\mathrm{H}$ at $3425 \mathrm{~cm}-1$ and a stretching vibration of $\mathrm{C}=\mathrm{O}$ at $1720 \mathrm{~cm}^{-1}$ [34]. The peak at $1580 \mathrm{~cm}^{-1}$ for $\mathrm{C}=\mathrm{C}$ stretching suggests skeletal vibrations of unoxidized graphitic domains or bending vibration of $\mathrm{H} 2 \mathrm{O}$ molecule present in $\mathrm{GO}$. The spectrum of GO also shows the $\mathrm{C}-\mathrm{O}$ stretching of epoxy and alkoxy groups at $1218 \mathrm{~cm}^{-1}$ and $1045 \mathrm{~cm}^{-1}$, respectively [33]. The spectrum of neat PPSU (Fig. 4b) contains sulfone, sulfonamide, and quinone groups. The sharp peaks at 1585 and $1486 \mathrm{~cm}^{-1}$ ascribed to the $\mathrm{C}-\mathrm{C}$ stretching of the aromatic rings, and those at 1684 and $1241 \mathrm{~cm}^{-1}$ are assigned to the $\mathrm{C}-\mathrm{O}$ stretching vibration of the ether group. The absorption bands at 1295 and $1151 \mathrm{~cm}^{-1}$ arise from the asymmetrical and symmetrical stretching vibrations of the $\mathrm{SO}_{2}$ group, respectively. In addition, the band at $1073 \mathrm{~cm}^{-1}$ is related to the $\mathrm{S}=\mathrm{O}$ stretching [46]. However, new features are seen in the spectra of the nanocomposite membranes (Figs. $4 \mathrm{c}$ and 4d) compared to the neat PPSU membrane (Fig. 4b). From the spectra a new peak at $3640 \mathrm{~cm}^{-1}$ can be observed, corresponding to the $-\mathrm{OH}$ vibration and a peak at $1779 \mathrm{~cm}^{-1}$ assigned to stretching vibration of $\mathrm{C}=\mathrm{O}$ and with increasing the loading of $\mathrm{GO}$, these peaks also increase. Appearance of these characteristic peaks confirms the existence of GO on the surface of the nanocomposite membranes.

As shown in Fig. 5, the PPSU/GO membranes obtained with different GO concentrations show uniform colors, which gradually change from white to grey with the increase of GO concentration, indicating that GO nanoplates are dispersed in to the polymer matrix homogeneously. 
The XRD patterns of GO, neat PPSU and the PPSU/GO with 0.1 wt. \% loading of GO are shown in Fig. 6. XRD is an important tool to shows dispersion of graphene-based plates in nanocomposites [47]. The characteristic peak of GO was observed at $2 \theta=12.7^{\circ}$ (Fig. 6a), and the diffraction peak of the neat PPSU membrane appeared at $2 \theta=18^{\circ}$ (Fig. 6b). However, after GO was dispersed into the PPSU matrix, the XRD pattern of the PPSU/GO nanocomposite (Fig. 6c) only showed the PPSU diffraction peak. The disappearance of the diffraction peak of GO indicates a high level of dispersion and exfoliation of the GO layers into the polymer matrix [47,48]. We can also observe from Fig. $6 c$ that the diffraction peak of the PPSU shifted slightly to the higher angle $\left(2 \theta=18.5^{\circ}\right)$. This shift is ascribed to the interaction between the polymer matrix and GO, giving rise to a reduction of the distance between polymer chains [49].

\subsubsection{Morphology of the nanocomposite support membranes}

The cross-sectional SEM images of the support membranes with different GO contents are presented in Fig. 7. All of the membranes show typical asymmetric structure consisting of a dense top layer followed by macro-void sub layer. GO embedded membranes demonstrated obvious changes in both top layer and sub layer. The micro-void pores for 0.05 and $0.1 \mathrm{wt}$. \% GO embedded membranes are slightly larger than that of the neat PPSU membrane. In addition, the thickness of the top layer decreases with increasing GO content to $0.1 \mathrm{wt} . \%$ and the fingerlike pores become closer to the top layer of the membrane. The addition of hydrophilic GO to polymer solution could increase the mass transfer rate between the solvent and non-solvent and thus favor pore formation [35,50]. Also some lateral pore structures appeared when the GO concentration increased from $0 \mathrm{wt}$. \% to $0.1 \mathrm{wt}$. \%, similar to the fabrication of other GO blended membranes $[33,35,50]$. These features improved the water fluxes of the membranes. On the other hand, the addition of excess GO influenced the thickness of the top layer and the pore structure of the porous layer as shown in Fig. 7. The support membranes with GO content over 0.1 wt. \% have a thicker top layer and smaller micro-void pores. This can be ascribed to the kinetic hindrance due to an increase in polymer solution viscosity after more GO is added. The increase of the viscosity delays the mass transfer rate between the solvent and non-solvent as well as prevent the formation of larger finger-like voids. This was also returned by the lower porosity at $\mathrm{GO} \geq 0.2$ wt. \% than those at lower concentration (Table 4). The overall porosities and mean pore radius of the nanocomposite support membranes are summarized in Table 4 . The mean pore 
size and overall porosity of the membranes are initially increased with the addition of a small content of GO and then decreased. This is attributed to the viscosity of the casting solutions, which delayed the membrane-forming process.

The support membranes were studied for their surface roughness using AFM measurements. It was found that GO nanoplates play a role in affecting support surface roughness as shown in Fig. 8. The surface roughness parameters of the support membranes are presented in Table 5. The roughness properties of the PPSU/GO decreased as GO loading was increased to $0.1 \mathrm{wt}$. \% and then increased again at $\geq 0.2$ wt. \% loadings. This shows that well-dispersed GO could smoothen the surface of PPSU/GO, which was significant in $0.1 \mathrm{wt}$ \% GO. The same behavior has been reported in previous studies [28,50-52]. In low loading of carbon-based nanofiller, because of low electrostatic interaction and good compatibility with the membrane matrix, these nanomaterials are generally collocated in membrane and create a smooth surface [53]. This characteristic could be most favorable for the formation of PA layer by IP technique, which positively influences the rejection performance of TFN-NF membrane [54]

\subsubsection{PWF and hydrophilicity}

The PWF and contact angle of the PPSU/GO membranes are given in Table 4. The hydrophilicity of the membrane can be studied by water contact angle measurements. The contact angle of the mixed membranes decreased with the addition of GO nanoplates, indicating that the modified substrates are more hydrophilic than the unmodified substrate. This behavior can be ascribed to the fact that the hydrophilic GO nanoplates migrate to the membrane surface during the phase inversion process to reduce the interface energy [32,52] This is clear from the FTIR spectra of the membranes (Fig. 4) which confirmed the presence of hydrogen- bonded hydroxyl group. Also this mechanism can be shown by the different color between the top and bottom surfaces- the top surface was darker than the bottom surface. Similar results were also reported for the GO-PSf [32] and GO-PES [50] mixed matrix membranes. However, when the amount of GO was increased to 0.4 wt. \%, the water contact angle of the membrane increased slightly. This is possibly due to agglomeration and irregular position of GO in the membrane surface at over $0.2 \mathrm{wt}$. \% GO content, which comes to decrease of the membrane surface functional groups [50,52]. 
As indicated in Table 4, with increasing GO content, the PWFs of the blend membranes were improved, and mainly supported by the increase of surface pore size, porosity, and hydrophilicity. The PWFs of the PPSU/GO substrates increased first with increasing GO content and then decreased (over $0.1 \mathrm{wt}$ \%), similar to the variation tendency of surface porosity.

\subsection{Characterization of the TFN-NF membrane}

The chemical characterization of the NF membranes was done by ATR-IR spectroscopy. Fig. 9 illustrates the ATR-IR spectra of the pure PPSU support, TFC, and TFN(0.1) membranes made of different types of supports. A comparison between the support and TFC and TFN spectrums revealed that the IP process between PIP and TMC was successfully performed over both pure and nanocomposite supports, as a strong broad band at $1614 \mathrm{~cm}^{-1}$, which corresponds to the $\mathrm{C}=\mathrm{O}$ band of an amide group, was found for the TFN and TFC membranes.

The FESEM and AFM analyses were conducted to investigate the morphological structure of PA layer, which was interfacially polymerized over the PPSU and PPSU/GO substrates. The top surface and cross-sectional FESEM images for PA selective layers built on different supports are shown in Fig. 10. As indicated, the surface morphology of PA polymerized by PIP and TMC has "ridge and valley" and "nodular" structure, whereas, the surface of the TFN membranes formed over PPSU/GO blend supports are more "nodular". The reason for the difference in the surface morphology between the TFN and TFC membranes is likely related to the chemistry and structure of the support as explained by other researchers [14,27,55]. When using nanocomposite support membranes, the diffusion rate of PIP from inside pore to membrane interface would be certainly slow due to favorable hydrogen bond interactions with the hydrophilic support, limiting the size of initial polyamide tufts, and giving "nodular' morphology. Increasing GO content from 0.05 to 0.2 wt. \% in the support made toward a combination of "ridge and valley" and "nodular" structure in the PA layer surface, which indicates the formation of a dense and crosslinked structure. When GO content was above 0.2 wt. \%, the surface of the PA layer was smooth with "nodular" structure.

The thickness of the NF membranes was measured at three different locations for each sample. Average calculated values of thin film thickness $\left(\delta_{\text {film }}\right)$ are reported in Fig. 10. According to the 
cross-sectional images (Fig.10), there was a substantial difference in the PA layer thickness. With increasing GO content in the support, the thickness of the PA layer initially decreased and then increased (over 0.1 wt. \%). The pore size of the support as well as hydrophilicity affects the diffusion of PIP through the pore and formation of PA layer [14-16,20,22,56]. Larger pores of the support favor the penetration of amine monomer into the pores, leading to formation of polyamide film inside the pore and form polyamide thinner with a longer effective film thickness for water permeation. According to the results obtained from the measurement of pore size and porosity of the support membranes (Table 4), it is expected to form a thinner PA layer over the 0.1 wt. \% GO embedded support membrane.

Since the parameters of IP are constant during the synthesis of the PA thin layer, the physicochemical properties of substrates play a significant role in the modifications of surface morphology, and thickness of the PA layer. Fig. 11 shows the AFM topographic images together with roughness values of the top surface of TFC and TFN $(0.1)$ membranes. Clearly, the surface of TFC membrane was much rougher compared to the TFN membrane. The $\mathrm{R}_{\mathrm{a}}$ value of TFC membrane was around $4.49 \mathrm{~nm}$ compared to $3.43 \mathrm{~nm}$ shown in TFN(0.1) membrane. Apart from the effect of pore size of the PPSU/GO support, the slight decrease in support roughness upon addition of GO is also found to cause the surface roughness of the TFN membrane to decrease [57].

\subsection{Performance evaluation of TFC and TFN membranes}

\subsubsection{PWF and salt rejection}

The PWF and $\mathrm{MgSO}_{4}$ rejection were measured using a cross-flow NF experimental setup to evaluate separation performance of the prepared NF membranes. Fig. 12 presented the water flux and salt rejection of the TFN and TFC membranes. The rejection of $\mathrm{MgSO}_{4}$ was over $90 \%$ and the salt rejection of the TFN membranes was slightly decreased from $92.5 \%$ to $90.5 \%$. It is supposed that the decrease in the salt rejection is due to the lowering of PA cross-linking, which is likely caused by the porous structure and/or the GO agglomeration on the PPSU/GO support. It was also observed from Fig. 12 that the flux increases with increasing GO content in the support and then starts decreasing (over 0.1 wt. \%). Based on the FESEM image analysis (Fig. $10)$, $\mathrm{TFN}(0.1)$ membrane is thinner than TFC membrane, hence high water flux was generated 
by the thinner membrane. The water flux of TFN $(0.1)$ membrane was shown to be $46\left(\mathrm{~L} / \mathrm{m}^{2} . h\right)$, whereas the flux of TFC membrane was $40.3\left(\mathrm{~L} / \mathrm{m}^{2} . h\right)$. This flux enhancement can be explained by the improved hydrophilicity of the support layer together with the increased overall porosity upon addition of GO as shown in Table 4.

\subsubsection{Removal of the KHI from water}

The performance of TFC and TFN(0.1) membranes in terms of permeate flux and rejection of KHI are presented in Figs. 13 and 14. NF experiments have been carried out for KHI aqueous solutions with concentrations in the range of $0.1-1 \mathrm{wt} . \%$ at different pressures. The permeate flux and KHI rejection versus the operating pressure for the two membranes are plotted in Fig. 13. It was determined that the permeate flux increases with the pressure and the higher fluxes are obtained for the TFN(0.1) membrane. Whereas for the pressure- driven membrane process, the flux depends on the pressure across the membrane, increasing the operating pressure increases the pressure and consequently the flux [58]. The membranes showed the KHI rejection higher than $97 \%$, and the rejection increased to $99.5 \%$ for TFC and $99 \%$ for $\operatorname{TFN}(0.1)$ membranes by increasing the pressure. The solute flux depends on concentration gradient across the membrane. Therefore, when the pressure increases, water is pushed through the membrane at a quicker rate than solute and rejection increases [58]. Fig. 14 shows the permeate flux and KHI rejection against the KHI concentration for TFC and TFN(0.1) membranes. The results show that the flux and KHI rejection across the membranes decreased when the KHI concentration in feed solution increased. Increasing feed concentration can efficiently increase the osmotic pressure in the solution and the overall membrane resistance, hence the flux decreased [59]. In addition, the solute passage across the membrane increases with increasing the feed concentration due to effect of concentration polarization and sorption of KHI on the surface of membrane [59]. Figs. 13 and 14 indicate that the prepared TFC and TFN membranes have potential for rejection of KHI from aqueous solution and the TFN membrane have a higher flux, which means that the performance of the membrane was improved by addition of GO nanoplates. As mentioned in the previous section, due to the helpful effects of the GO on the structure of support layer and consequently the formation of PA selective layer, the flux of TFN(0.1) membrane is higher than that of TFC membrane. 


\subsubsection{Antifouling properties}

Membrane fouling resistance to the KHI was investigated through cross-flow fouling experiments employing TFC and TFN(0.1) membranes. However, the decrease of permeate flux or irreversible membrane fouling is a major challenge in efficient application of membrane filtration technologies [60]. To understand more about the fouling behavior, the membranes fouling was calculated from the data shown in Fig. 15 through eqns (5)- (7) presented in Table 3. Fig. 15 compares the flux trends before and after KHI filtration for TFC and TFN(0.1) membranes as a function of time. The flux of the membranes decreased greatly when pure water was replaced by the KHI solution in the filtration cell, which indicates the fouling process occurred on the membranes. The flux decline analysis and FRR of the prepared membranes was calculated and is listed in Table 6. The total fouling of the TFN membrane is lower than that of TFC membrane. Flux decline caused by the irreversible fouling for the TFN membrane was about $10 \%$ and the reversible fouling was the larger contribution to the total fouling, which shows that fouling was not very severe. The FRR of the TFN membrane is obviously higher than TFC membrane, which indicates the high antifouling property of the TFN membrane. The improved antifouling properties of the TFN membrane can be attributed to the improved smoothness of the membrane surface as shown in Fig. 11. Contaminants are likely to be absorbed in the valleys of the membrane; thus antifouling can be improved by an increase in the membrane surface smoothness [52]. Therefore, the TFN membrane with GO in the support layer could be used as a suitable membrane in the filtration of industrial effluents and treatment of wastewaters. Similar results were also reported for the TFC membrane with carbon nanotube in the PES support layer [61].

\section{Conclusion}

In the present study, new TFN-NF membranes were developed through IP method on the PPSU/GO supports. It can be concluded that the properties of the PPSU support was successfully modified by adding different amounts of GO nanoplates into the casting solution and the nanocomposite support effectively enhanced the NF performance of the TFN membrane. The surface pore size, porosity, hydrophilicity, and PWF of the PPSU/GO nanocomposite support 
was improved upon addition of GO, and the optimum concentration of GO in the casting solution was 0.1 wt. \%. The addition of GO in the support layer was found to have a significant effect on the surface morphology, thickness, and surface roughness of the PA layer. Among the TFN membranes prepared in this work, TFN $(0.1)$ membrane (prepared from PPSU blended with 0.1 wt. \% GO) was found to be the best performing NF membrane due to high water flux and good $\mathrm{MgSO}_{4}$ rejection. The performance of $\mathrm{TFN}(0.1)$ and TFC membranes was investigated by rejection of the KHI under different operating pressures and feed concentrations. The results indicated that the TFN membrane has a higher permeate flux compared to the TFC membrane, while the KHI rejection was almost the same. The permeate flux and KHI rejection increased with increased pressure and decreased with increasing of the feed concentration. The antifouling performance of TFC and TFN membranes was investigated with the KHI aqueous solution. The total flux decline of the TFN membrane was lower than TFC membrane and the FRR of the TFN membrane was noticeably higher. The overall results in this study demonstrate that the GOmodified PPSU support layer could be a favorable technique to produce TFN-NF membranes with significantly improved water flux and antifouling properties.

\section{Acknowledgements}

The authors gratefully acknowledge Iran National Science Foundation (INSF) and Iran Nanotechnology Initiative Council for financial support.

\section{Nomenclature}

\begin{tabular}{|cl|}
\hline KHI & Kinetic Hydrate Inhibitor \\
TFC & Thin film composite \\
TFN & Thin film nanocomposite \\
NF & Nanofiltration \\
PPSU & Polyphenylsulfone \\
GO & Graphene oxide \\
PA & Polyamide \\
PWF & Pure water flux \\
\hline
\end{tabular}




\begin{tabular}{|c|c|}
\hline UF & Ultrafiltration \\
\hline RO & Reverse osmosis \\
\hline IP & Interfacial polymerization \\
\hline PVDF & Polyvinylidene fluoride \\
\hline PES & Polyethersulfone \\
\hline PSf & Polysulfone \\
\hline FTIR & Fourier transform infrared \\
\hline ATR-FTIR & $\begin{array}{l}\text { Attenuated total reflectance Fourier transform infrared } \\
\text { spectroscopy }\end{array}$ \\
\hline XRD & X-ray diffraction \\
\hline SEM & Scanning electron microscopy \\
\hline FESEM & Field emission scanning electron microscopy () and \\
\hline AFM & Atomic force microscopy \\
\hline $\mathbf{R}_{\mathbf{a}}$ & Average roughness \\
\hline $\mathbf{R}_{\mathbf{q}}$ & root mean square of the $\mathrm{Z}$ data \\
\hline PIP & Piperazine \\
\hline TMC & Trimesoyl chloride \\
\hline DI & Deionized \\
\hline$\varepsilon$ & Overall porosity (\%) \\
\hline$\omega$ & Wet membrane weight $(\mathrm{kg})$ \\
\hline$\omega$ & Dried membrane weight $(\mathrm{kg})$ \\
\hline $\mathbf{A}$ & Surface area of the membrane $\left(\mathrm{m}^{2}\right)$ \\
\hline$l$ & Membrane thickness (m) \\
\hline$\rho w$ & Water density $\left(\mathrm{kg} / \mathrm{m}^{3}\right)$ \\
\hline$\eta$ & Water viscosity (Pa. s) \\
\hline $\mathbf{Q}$ & volume of permeated pure water per unit time $\left(\mathrm{m}^{3} / \mathrm{s}\right)$ \\
\hline$\Delta \mathbf{P}$ & Transmembrane pressure (bar) \\
\hline $\mathbf{r}_{\mathrm{m}}$ & Mean pore radius (nm) \\
\hline
\end{tabular}




\begin{tabular}{|cl|}
\hline $\mathbf{V}$ & Permeate volume (L) \\
$\mathbf{t}$ & Permeate collection time (h) \\
$\mathbf{J}$ & Flux $\left(\mathrm{L} / \mathrm{m}^{2} . \mathrm{h}\right)$ \\
$\mathbf{R}$ & Rejection $(\%)$ \\
$\mathbf{C}_{\mathbf{p}}$ & Permeate concentrations \\
$\mathbf{C}_{\mathbf{f}}$ & Feed concentrations \\
$\mathbf{F R R}$ & Flux recovery ratio (\%) \\
\hline
\end{tabular}

\section{References}

[1] F-R. Ahmadun, A. Pendashteh, L. C. Abdullah, D. Radiah Awang Biak, S. S. Madaeni, Z. Zainal Abidin, Review of technologies for oil and gas produced water treatment, Journal of Hazardous Materials, 170 (2009) 530-551.

[2] E. D. Sloan Jr., C. Koh, Clathrate Hydrates of Natural Gases, Third ed., CRC Press, Taylor \& Francis Group, Boca Raton, Florida, 2008.

[3] M. A. Kelland, Production Chemicals for the Oil and Gas Industry, second ed., CRC Press, Taylor \& Francis Group, Boca Raton, Florida, 2014.

[4] L. Del Villano, M. A. Kelland, An investigation into the laboratory method for the evaluation of the performance of kinetic hydrate inhibitors using superheated gas hydrates, Chemical Engineering Science, 66 (2011) 1973-1985.

[5] D. J. Blumer, Y. Xu, B. B. Randolph, J. A. Cruze, Water impurity removal methods and systems, Patent application, US20130087502 A1, 2013.

[6] L. D. Talley, K. S. Colle, Recovery of kinetic hydrate inhibitor, Patent application, US7994374 B2, 2008.

[7] S. Adham, S. Gharfeh, A. Hussain, J. Minier Matar, A. Janson, Kinetic Hydrate Inhibitor removal by physical, chemical and biological processes, Offshore Technology ConferenceAsia, 25-28 March, Kuala Lumpur, Malaysia , 2014. 
[8] A. Hussain, I. Riza Turkmen, J. Minier Matar, S. Gharfeh, S. Adham, Advanced oxidation of kinetic hydrate inhibitors, Patent application, US20120241387, 2012.

[9] J. Minier Matar, I. Riza Turkmen, S. Gharfeh, S. Adham, A. Hussain, Removal of field chemicals from produced water using different membrane processes and system development, Patent application, US20130168315 A1, 2013.

[10] G. A. Schrader, Process for separating kinetic hydrate polymer inhibitors, Patent application, US20140144810 A1, 2014.

[11] G. A. Schrader, Process for separating kinetic hydrate polymer inhibitors, Patent application, EP 2433702 A1, 2012.

[12] A. Rahimpour, M. Jahanshahi, N. Mortazavian, S. S. Madaeni, Y. Mansourpanah, Preparation and characterization of asymmetric polyethersulfone and thin-film composite polyamide nanofiltration membranes for water softening, Applied Surface Science 256 (2010) 1657-1663.

[13] J.E. Cadotte, M.J. Steuck, R.J. Petersen, Research on in-situ-formed condensation polymers for reverse osmosis membranes, NTIS Rep. PB-288387, March, 1978.

[14] A.K. Ghosh, E.M.V. Hoek, Impacts of support membrane structure and chemistry on polyamide-polysulfone interfacial composite membranes, Journal of Membrane Science, 336 (2009) 140-148.

[15] N. Misdan, W.J. Lau, A.F. Ismail, T. Matsuura, D. Rana, Study on the thin film composite poly(piperazine-amide) nanofiltration membrane: Impacts of physicochemical properties of substrate on interfacial polymerization formation, Desalination, 344 (2014) 198-205.

[16] J. Xu, X. Feng, C. Gao, Surface modification of thin-film-composite polyamide membranes for improved reverse osmosis performance, Journal of Membrane Science, 370 (2011) 116123.

[17] M. Peyravi, A. Rahimpour, M. Jahanshahi, Thin film composite membranes with modified polysulfone supports for organic solvent nanofiltration, Journal of Membrane Science, 423 (2012) 225-237. 
[18] K. Yoon, B. S. Hsiao, B. Chu, High flux nanofiltration membranes based on in- terfacially polymerized polyamide barrier layer on polyacrylonitrile nanofibrous scaffolds, Journal of Membrane Science, 326 (2009) 484-492.

[19] G. Z. Ramon, M. C. Y. Wong, E. M. V. Hoek, Transport through composite membrane, Part1: Is there an optimal support membrane?, Journal of Membrane Science, 415-416 (2012) 298-305.

[20] P.S. Singh, S.V. Joshi, J.J. Trivedi, C.V. Devmurari, A. PrakashRao, P.K. Ghosh, Probing the structural variations of thin film composite RO membranes obtained by coating polyamide over polysulfone membranes of different pore dimensions, Journal of Membrane Science, 278 (2006) 19-25.

[21] L. Huang, J.R. McCutcheon, Impact of support layer pore size on performance of thin film composite membranes for forward osmosis, Journal of Membrane Science, 483 (2015) 2533.

[22] S. Zhu, S. Zhao, Z. Wang, X. Tian, M. Sh, J. Wang, S. Wang, Improved performance of polyamide thin-film composite nanofiltration membrane by using polyetersulfone/polyaniline membrane as the substrate, Journal of Membrane Science, 493 (2015) 263-274.

[23] X. Liu, H. Yong Ng, Fabrication of layered silica-polysulfone mixed matrix substrate membrane for enhancing performance of thin-film vomposite forward osmosis membrane, Journal of Membrane Science, 481 (2015) 148-163.

[24] N. Ma, J. Wei, S. Qi, Y. Zhao, Y. Gao, C. Y. Tang, Nanocomposite substrates for controlling internal concentration polarization in forward osmosis membranes, Journal of Membrane Science, 441 (2013) 54-62.

[25] D. Emadzadeh, W. J. Lau, T. Matsuura, M. Rahbari-Sisakht, A. F. Ismail, A novel thin film composite forward osmosis membrane prepared from $\mathrm{PSf}-\mathrm{TiO}_{2}$ nanocomposite substrate for water desalination, Chemical Engineering Journal, 237 (2014) 70-80.

[26] Y. Wang, R. Ou, Q. Ge, H. Wang, T. Xu, Preparation of polyethersulfone/carbon nanotube substrate for high-performance forward osmosis membrane, Desalination, 330 (2013) 70-78. 
[27] M. Namvar-Mahboub, M. Pakizeh, Development of a novel thin film composite membrane by Interfacial polymerization on polyetherimide/ modified $\mathrm{SiO} 2$ support for organic solvent nanofiltration, Separation and Purification Technology, 119 (2013) 35-45.

[28] M. Jun Park, S. Phunstho, T. He, G. M. Nisola, L. D. Tijing, X-M. Li, G. Chen, W-J. Chung, H. Kyong Shon, Graphene oxide incorporated polysulfone substrate for the fabrication of flat-sheet thin-film composite forward osmosis membranes, Journal of Membrane Science, 493 (2015) 496-507.

[29] M. Ionita, A. Madalina Pandele, L. Crica, L. Pilan, Improving the thermal and mechanical properties of polysulfone by incorporation of graphene oxide, Composites: Part B, 59 (2014) $133-139$.

[30] F. Perreault, M.E. Tousley, M. Elimelech, Thin-film composite polyamide membranes functionalized with biocidal graphene oxide nanosheets, Environmental Science \& Technology Letters, 1 (2014) 71-76.

[31] J. Lee, H-R. Chae, Y. June Won, K. Lee, C-H. Lee, H. H. Lee, I. Kim, J-M. Lee, Graphene oxide nanoplatelets composite membrane with hydrophilic and antifouling properties for wastewater treatment, Journal of Membrane Science, 448 (2013) 223-230.

[32] B. M. Ganesh, A. M. Isloor, A. F. Ismail, Enhanced hydrophilicity and salt rejection study of graphene oxide-polysulfone mixed matrix membrane, Desalination, 313 (2013) 199-207.

[33] S. Zinadini, V. Vatanpour, A. A. Zinatizadeh, M. Rahimi, Z. Rahimi, M. Kian, Preparation and characterization of antifouling graphene oxide/polyethersulfone ultrafiltration membrane: Application in MBR for dairy wastewater treatment, Journal of Water Process Engineering, 7 (2015) 280-294.

[34] F. Jin, W. Lv, C. Zhang, Z. Li, R. Su, W. Qi, Q-H. Yang, Z. He, High-performance ultrafiltration membranes based on polyethersulfone-graphene oxide composites, RSC Advances, 3 (2013) 21394-21397.

[35] Z. Wang, H. Yu, J. Xia, F. Zhang, F. Li, Y. Xia, Y. Li, Novel GO-blended PVDF ultrafiltration membranes, Desalination, 299 (2012) 50-54. 
[36] C. Zhao, X. Xu, J. Chen, F. Yang, Effect of graphene oxide concentration on the morphologies and antifouling properties of PVDF ultrafiltration membranes, Journal of Environmental Chemical Engineering, 1 (2013) 349-354.

[37] M. Hu, B. Mi, Enabling graphene oxide nanosheets as water separation membranes, Environmental science \& technology, 47 (2013) 3715-3723.

[38] E. Mahmoudi ,L. Yong Ng ,M. M. Ba-Abbad , A. W. Mohammad, Novel nanohybrid polysulfone membrane embedded with silver nanoparticles on graphene oxide nanoplates, Chemical Engineering Journal, 277( 2015) 1-10.

[38] J. Zhang, Z. Xu, W. Mai, C. Min, B. Zhou, M. Shan, Y. Li, C. Yang, Z. Wang, X. Qian, Improved hydrophilicity, permeability, antifouling and mechanical performance of PVDF composite ultrafiltration membranes tailored by oxidized low dimensional carbon nanomaterials, Journal of Materials Chemistry A, 1 (2013) 3101-3111.

[40] Z. Xu, J. Zhang, M. Shan, Y. Li, B. Li, J. Niu, B. Zhou, X. Qian, Organosilanefunctionalized graphene oxide for enhanced antifouling and mechanical properties of polyvinylidene fluoride ultrafiltration membranes, Journal of Membrane Science, 458 (2014) $1-13$.

[42] Y. Liu, S. Zhang, Z. Zhou, J. Ren, Z. Geng, J. Luan, G. Wang, Novel sulfonated thin-film composite nanofiltration membranes with improved water flux for treatment of dye solutions, Journal of Membrane Science, 394- 395 (2012) 218- 229.

[43] N. Widjojo, T-S. Chung, M. Weber, C. Maletzko, V. Warzelhan, A sulfonated polyphenylenesulfone (sPPSU) as the supporting substrate in thin film composite (TFC) membranes with enhanced performance for forward osmosis (FO), Chemical Engineering Journal, 220 (2013) 15-23.

[44] M. Mulder, Basic Principles of Membrane Technology, Kluwer Academic Publishers, Dordrecht, 1996.

[45] S. Zinadini, A.A. Zinatizadeh, M. Rahimi, V. Vatanpour, H. Zangeneh, M. Beygzadeh, Novel high flux antifouling nanofiltration membranes for dye removal containing carboxymethyl chitosan coated $\mathrm{Fe}_{3} \mathrm{O}_{4}$ nanoparticles, Desalination, 349 (2014) 145-154. 
[46] A. M. Diez-Pascual, A. L. Diez-Vicente, Effect of $\mathrm{TiO}_{2}$ nanoparticles on the performance of polyphenylsulfone biomaterial for orthopaedic implants, Journal of Materials Chemistry B, 2 (2014) 7502-7514.

[47] J. Liang, Y. Huang, L. Zhang, Y. Wang, Y. Ma, T. Guo, Y. Chen, Molecular-level dispersion of graphene into poly(vinyl alcohol) and effective reinforcement of their nanocomposites, Advanced Functional Materials, 19 (2009) 2297-2302.

[48] S. Ansari, A. Kelarakis, L. Estevez, E. P. Giannelis, Oriented arrays of graphene in a polymer matrix by in situ reduction of graphite oxide nanosheets, Small, 6 (2010) 205-209.

[49] A.K. Zulhairun, A.F. Ismail, T. Matsuura, M.S. Abdullah, A. Mustafa, Asymmetric mixed matrix membrane incorporating organically modified clay particle for gas separation, Chemical Engineering Journal, 241 (2014) 495-503.

[50] S. Zinadini, A. A. Zinatizadeh, M. Rahimi, V. Vatanpour, H. Zangeneh, Preparation of a novel antifouling mixed matrix PES membrane by embedding graphene oxide nanoplates, Journal of Membrane Science, 453 (2014) 292-301.

[51] R. Rezaee, S. Nasseri, A. H. Mahvi, R. Nabizadeh, S. A. Mousavi, A. Rashidi, A. Jafari, S. Nazmara, Fabrication and characterization of a polysulfone-graphene oxide nanocomposite membrane for arsenate rejection from water, journal of Environmental Health Science \& Engineering, 13 (2015) 61-72.

[52] H. Zhao, L. Wu, Z. Zhou, L. Zhang, H. Chen, Improving the antifouling property of polysulfone ultrafiltration membrane by incorporation of isocyanate-treated graphene oxide, Physical Chemistry Chemical Physics, 15 (2013) 9084-9092.

[53] S. Qiu, L. Wu, X. Pan, L. Zhang, H. Chen, C. Gao, Preparation and properties of functionalized carbon nanotube/PSF blend ultrafiltration membranes, Journal of Membrane Science, 342 (2009) 165-172.

[54] Y. Wang, R. Ou, H. Wang, T. Xu, Graphene oxide modified graphitic carbon nitride as a modifier for thin film composite forward osmosis membrane, Journal of Membrane Science, 475 (2015) 281-289. 
[55] M. F. Jimenez Solomon, Y. Bhole, A. G. Livingston, High flux membranes for organic solvent nanofiltration(OSN) - Interfacial polymerization with solvent activation, Journal of Membrane Science, 423-424 (2012) 371-382.

[56] X. Li, K. Y. Wang, B. Helmer, T-S. Chung, Thin-film composite membranes and formation mechanism of thin-film layers on hydrophilic cellulose acetate propionate substrates for forward osmosis processes, Industrial \& Engineering Chemistry Research, 51 (2012) 1003910050.

[57] P. Veerababu, B. B. Vyas, P. S. Singh, P. Ray, Limiting thickness of polyamidepolysulfone thin-film-composite nanofiltration membrane, Desalination, 346 (2014) 19-29.

[58] R. W. Baker, Membrane Technology and Applications, Third ed., Membrane Technology and Research, John Wiley \& Sons, Ltd., Newark, California, 2012.

[59] S. Binyam, H. Mukhtar, K.K. Lau, Flux and Rejection of Methyldiethanolamine from Wastewater by Composite Reverse Osmosis Membrane, Journal of Applied Sciences, 10 (2010) 3331-3336.

[60] J. Mansouri, S. Harrisson, V. Chen, Strategies for controlling biofouling in membrane filtration systems: challenges and opportunities, Journal of Materials Chemistry, 20 (2010) $4567-4586$.

[61] M.Son, H. Choi, L. Liu, E. Celik, H. Park, H. Choi, Efficacy of carbon nanotube positioning in the polyethersulfone support layer on the performance of thin-film composite membrane for desalination, Chemical Engineering Journal, 266 (2015) 376-384. 


\section{Figures captions:}

Fig. 1. Scanning electron microscopy (SEM) image of GO nanoplates used in this study.

Fig. 2. Schematic illustration of TFC and TFN membrane formation.

Fig.3. The schematic diagram of cross flow system.

Fig. 4. FTIR spectra of the GO, neat PPSU and the PPSU/GO membranes with 0.1 and 0.4 wt.\% loading of GO.

Fig. 5. Digital photograph of the PPSU/GO membrane with different GO fractions.

Fig. 6. XRD patterns of the GO nanoplates, neat PPSU and the PPSU/GO with 0.1 wt. \% loading of GO.

Fig. 7. Cross-section SEM images of the PPSU/GO support membranes.

Fig. 8. AFM images of the PPSU/GO support membranes.

Fig. 9. ATR-IR spectra of the PPSU support, TFC and TFN (0.1) membranes.

Fig.10. The top surface and cross-sectional FESEM images of TFC and TFN membranes.

Fig. 11. AFM images of TFC and TFN (0.1) membranes.

Fig. 12. PWF and salt rejection of TFC and TFN membranes (test conditions: $P=8$ bar, $\mathrm{T}=25^{\circ} \mathrm{C}$ and $2000 \mathrm{ppm} \mathrm{MgSO}_{4}$ aqueous solution as feed).

Fig.13. Permeate flux and $\mathrm{KHI}$ rejection versus operating pressure for TFC and TFN (0.1) membranes (test conditions: $\mathrm{T}=25{ }^{\circ} \mathrm{C}$ and $\mathrm{C}_{\mathrm{f}}=0.5 \mathrm{wt} . \%$ ).

Fig. 14. Permeate flux and $\mathrm{KHI}$ rejection versus feed concentration for TFC and TFN (0.1) membranes (test conditions: $\mathrm{P}=8$ bar and $\mathrm{T}=25^{\circ} \mathrm{C}$ ).

Fig. 15. Flux versus time for the TFC and TFN (0.1) membranes at 8 bar. 
Figures:

Fig. 1

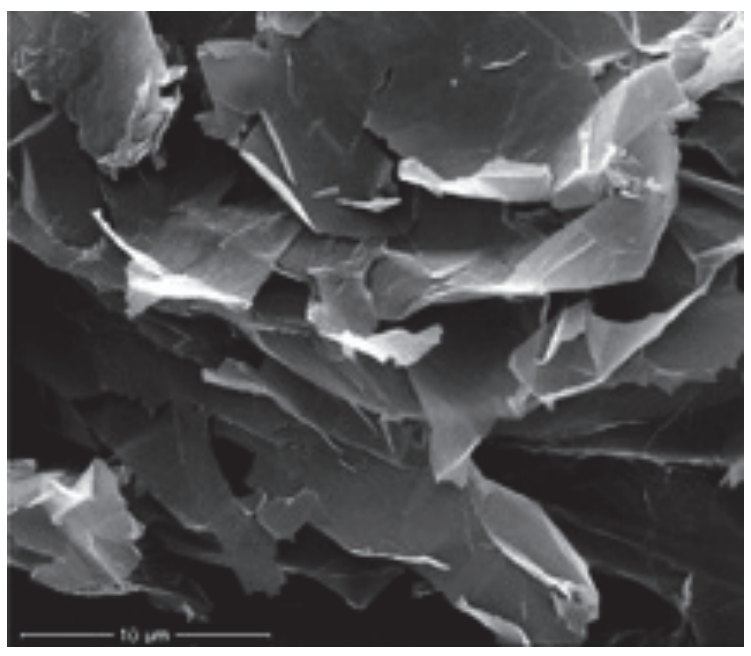


Fig. 2

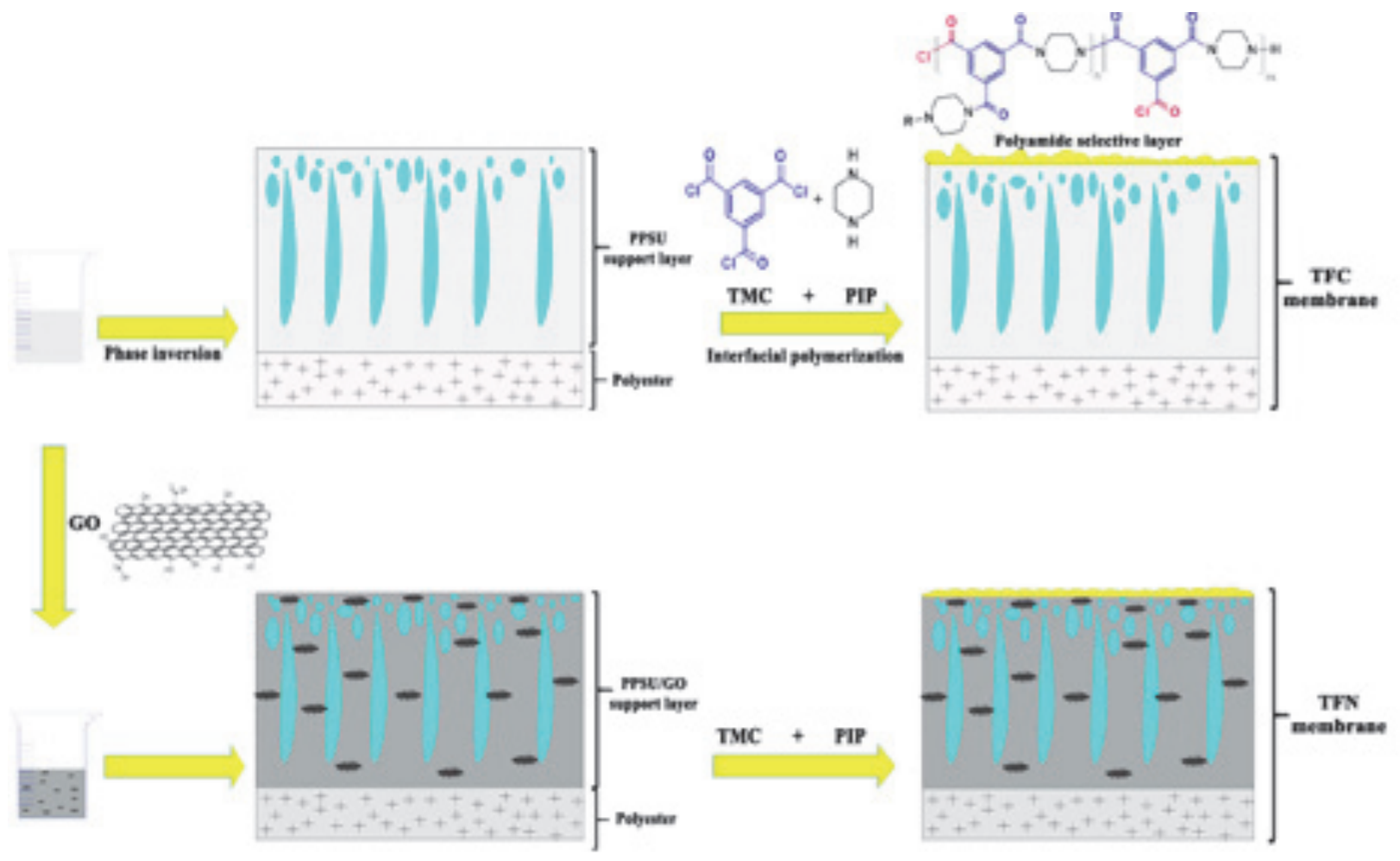


Fig.3

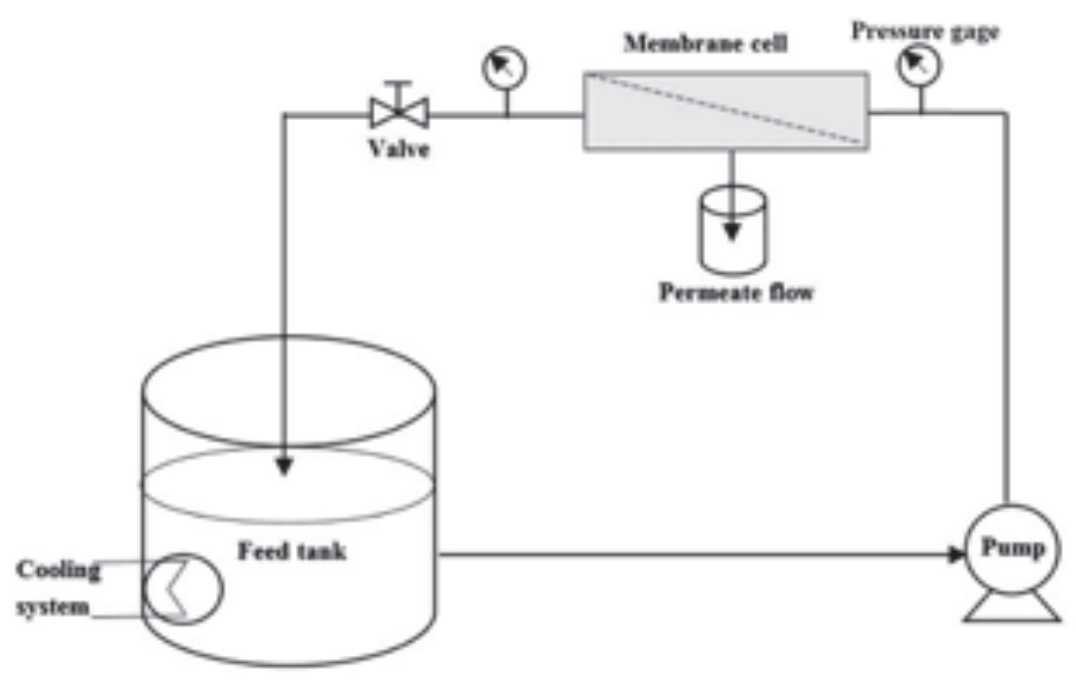


Fig. 4

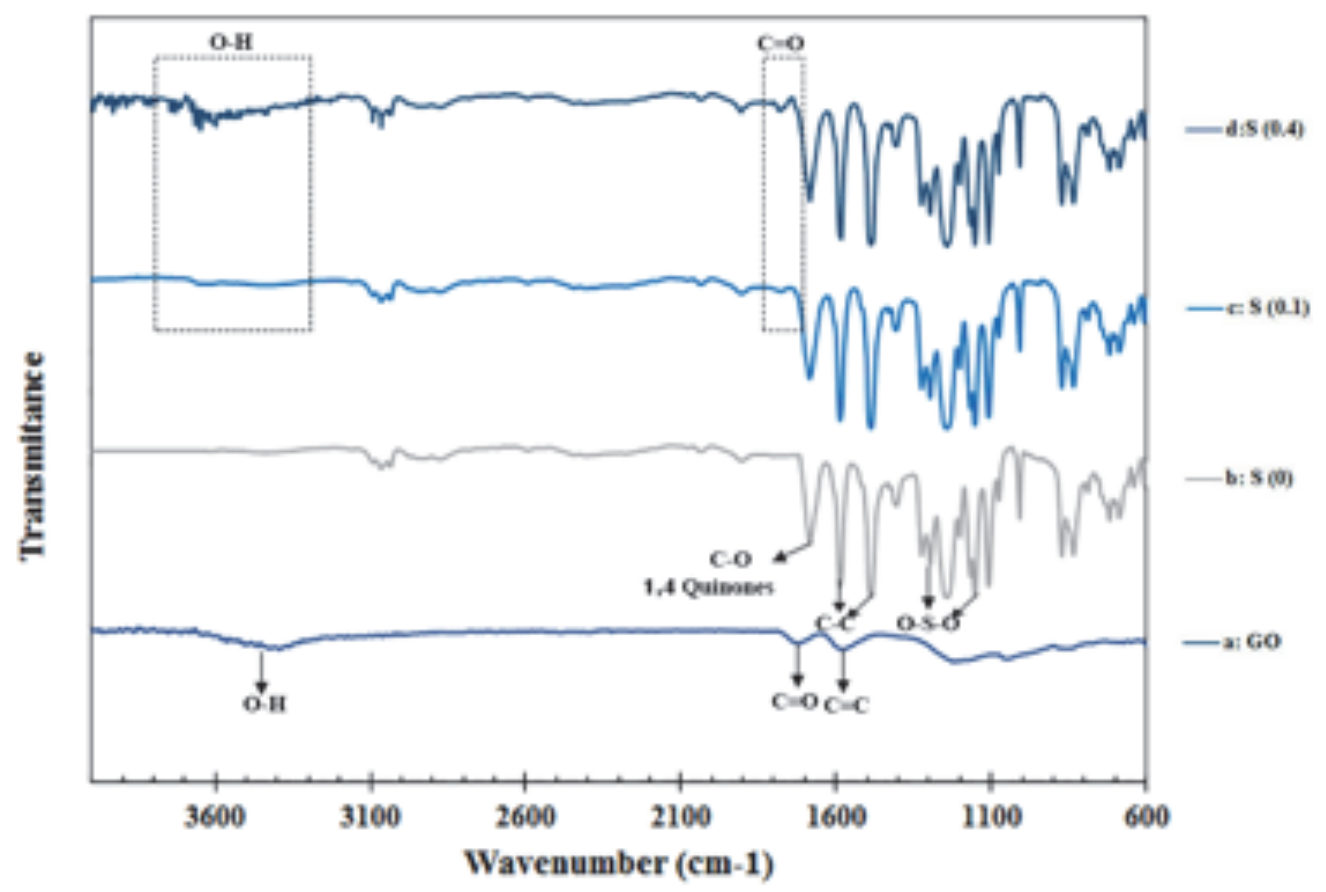


Fig. 5

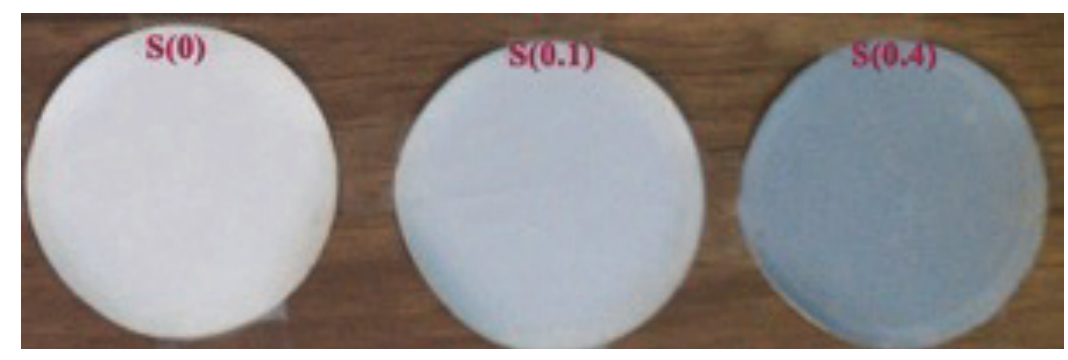


Fig. 6

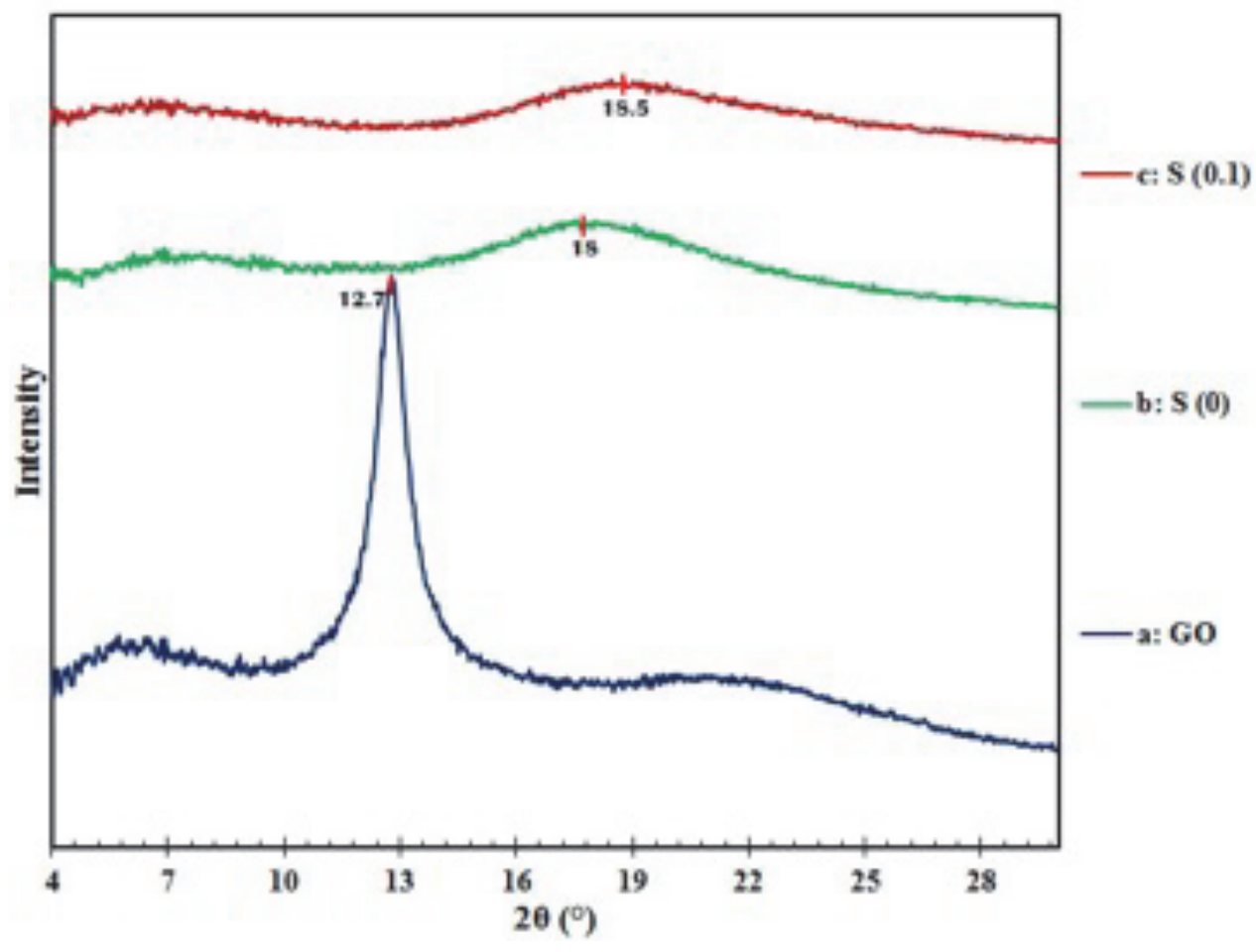


Fig. 7

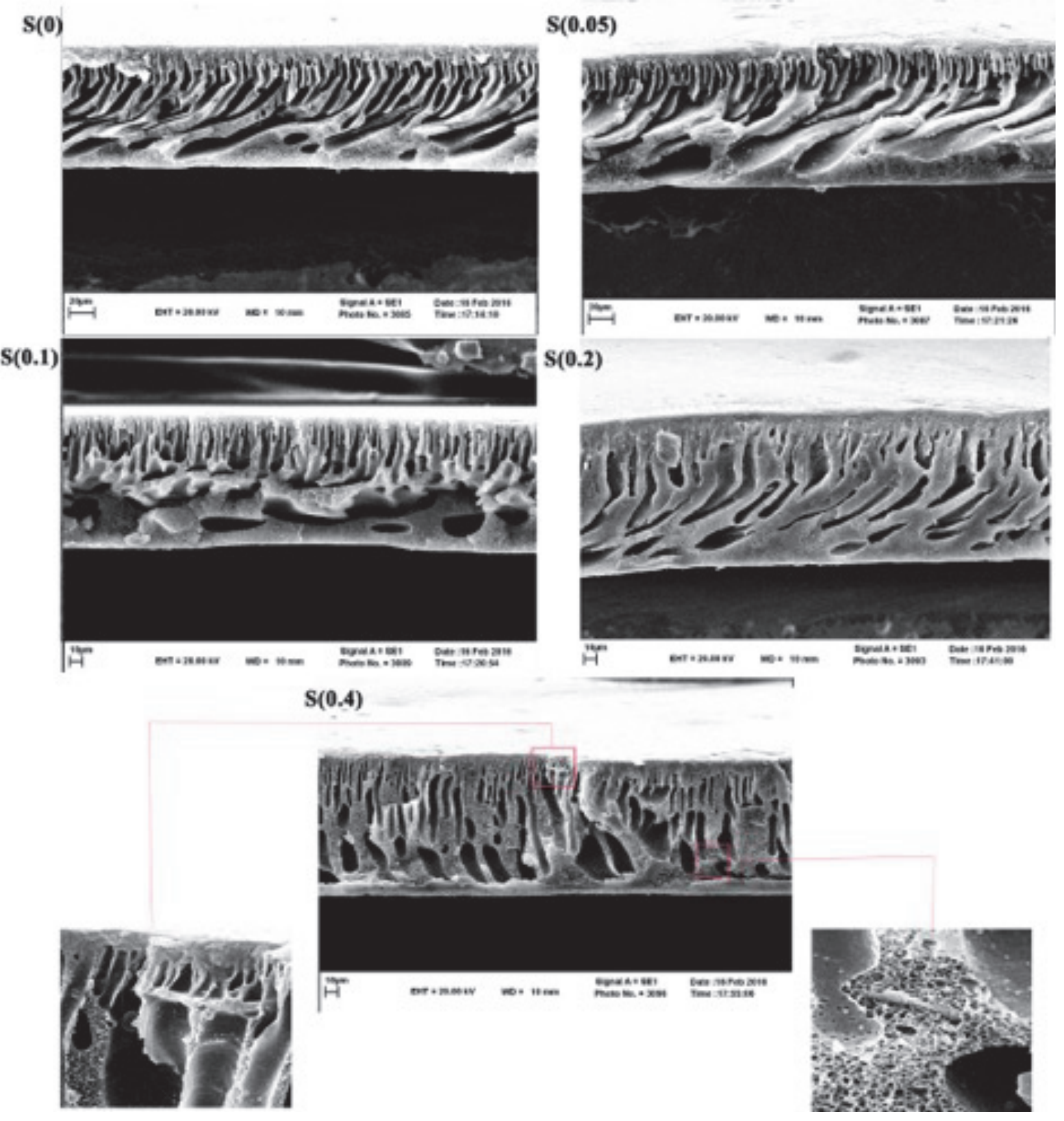


Fig. 8
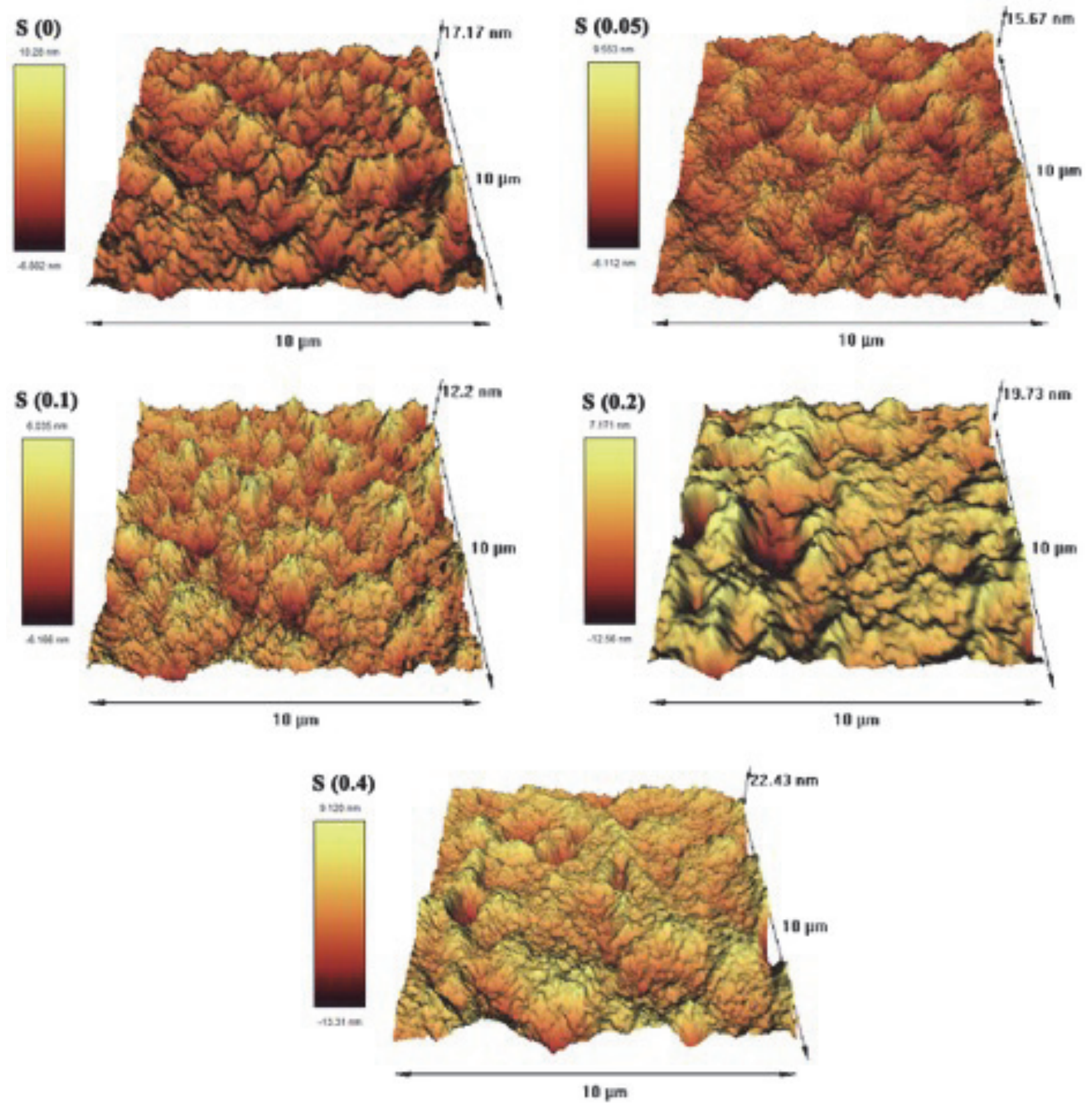
Fig. 9

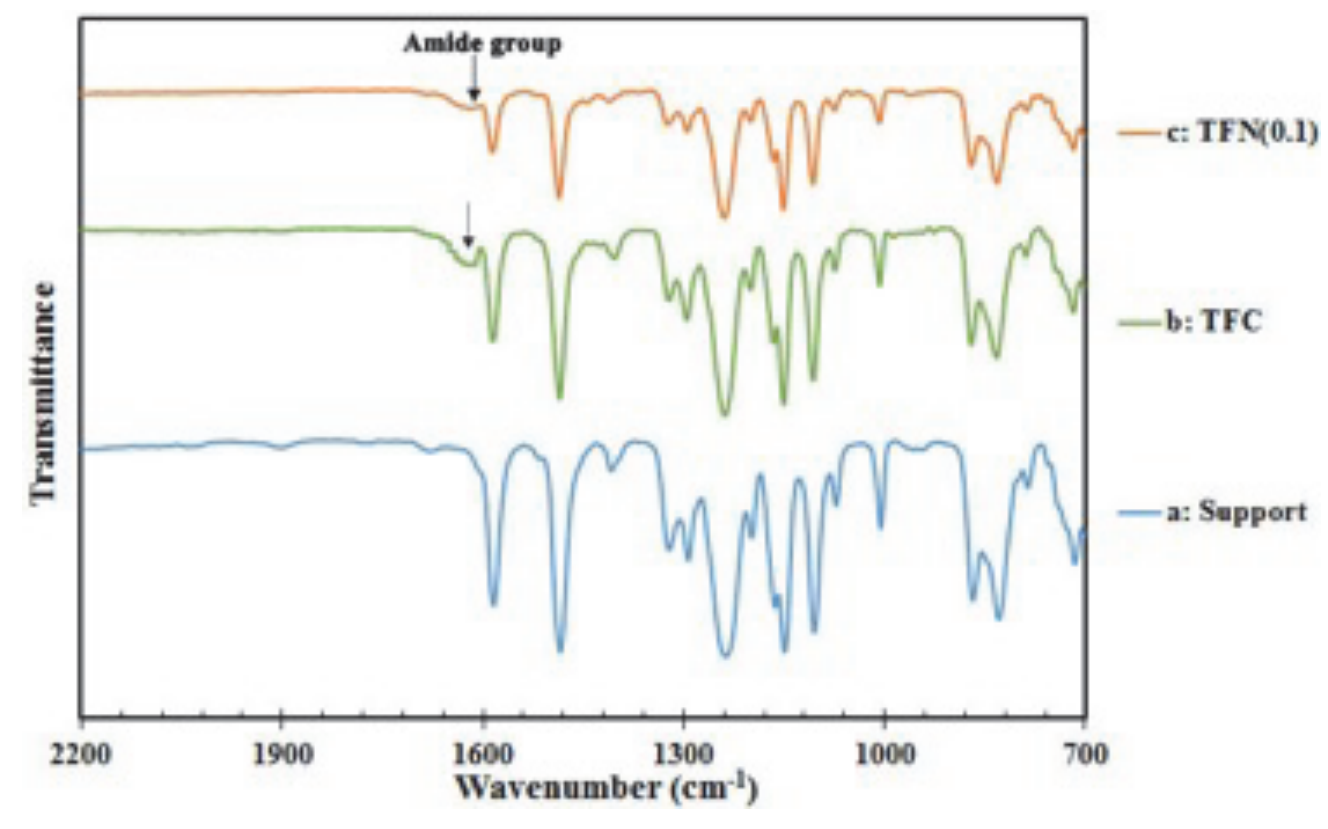


Fig.10
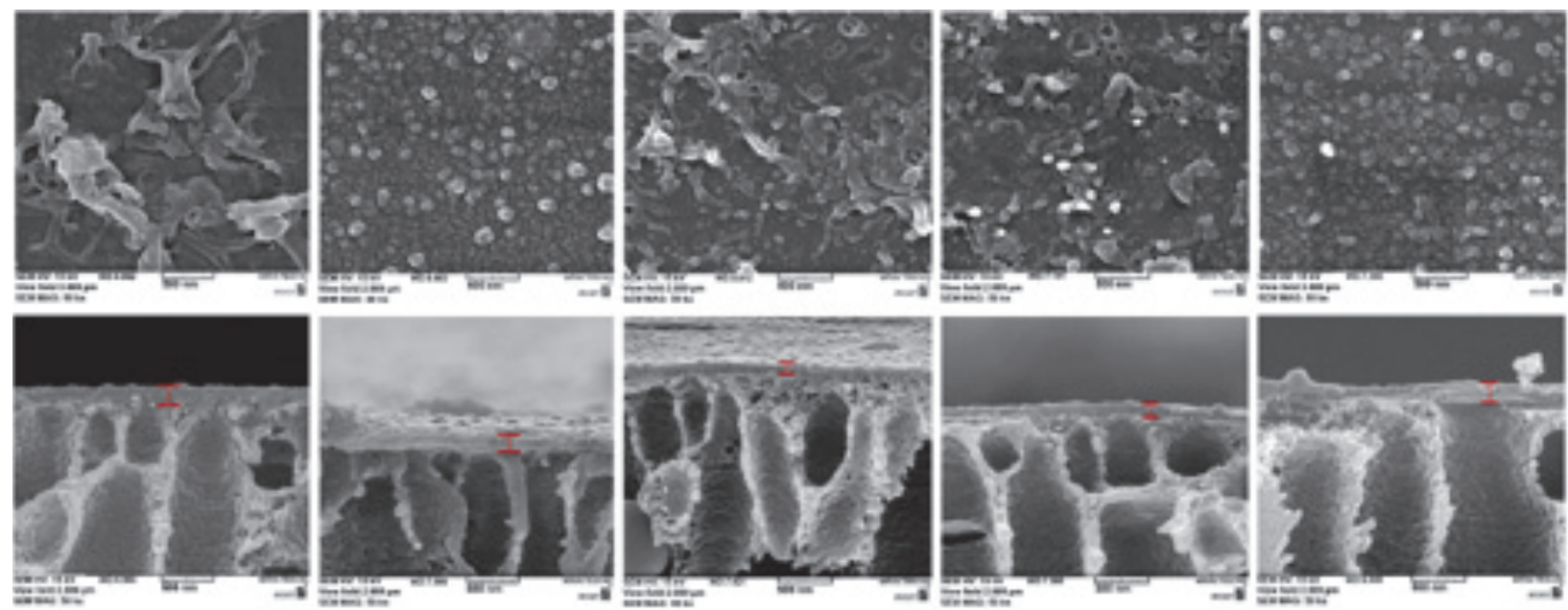

$\underset{\operatorname{Trc}}{\operatorname{mec}}$

TMNens

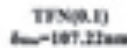

Mons.

monet 
Fig. 11

TFC

$R=4.49 \mathrm{~nm}$

$37.11 \mathrm{~mm}$

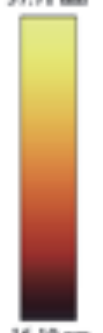

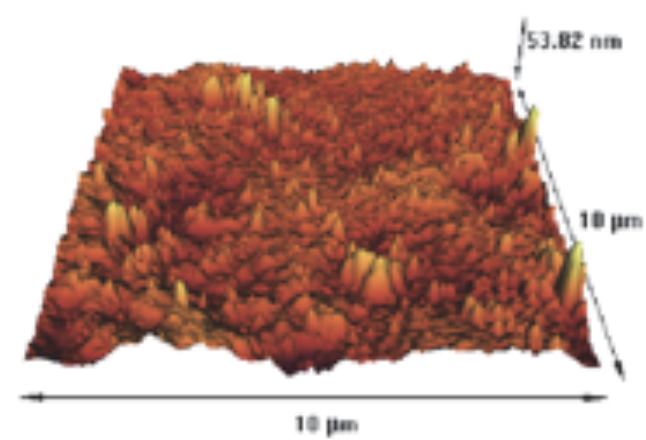

TFN(0.1)

$\mathrm{R}=3.43 \mathrm{~nm}$

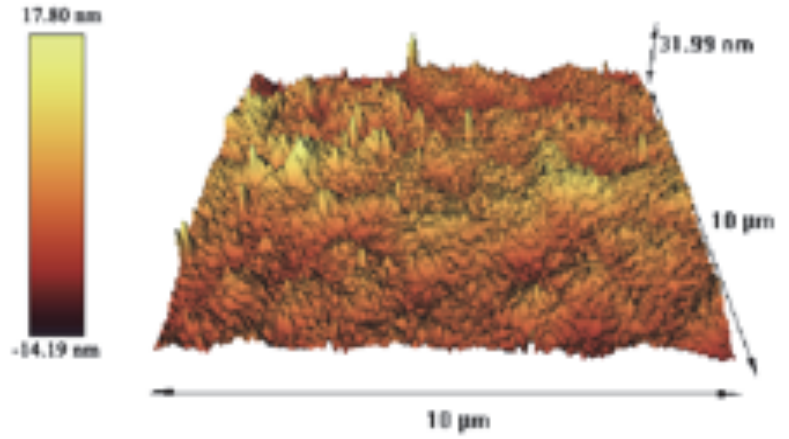


Fig. 12

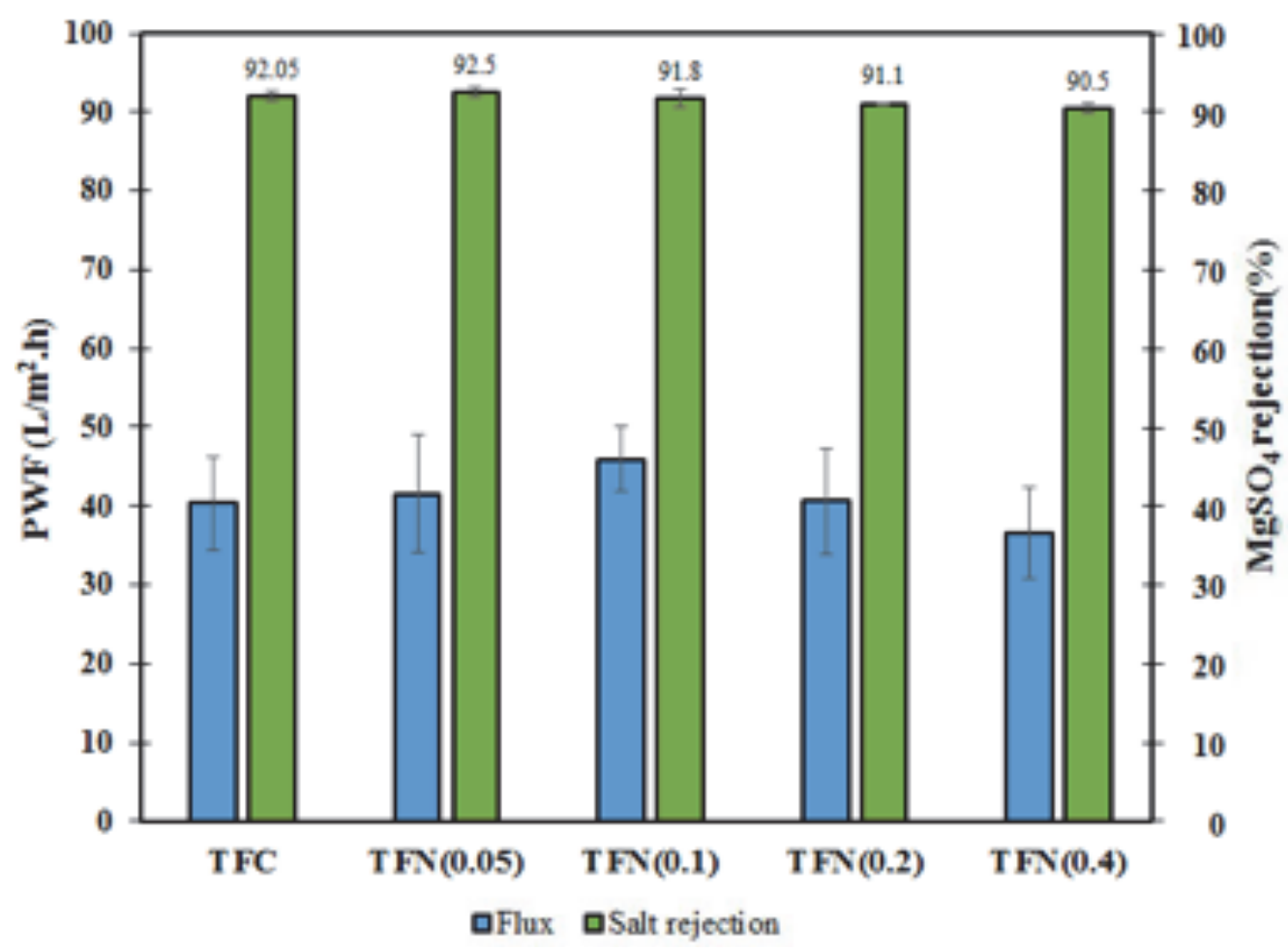


Fig.13

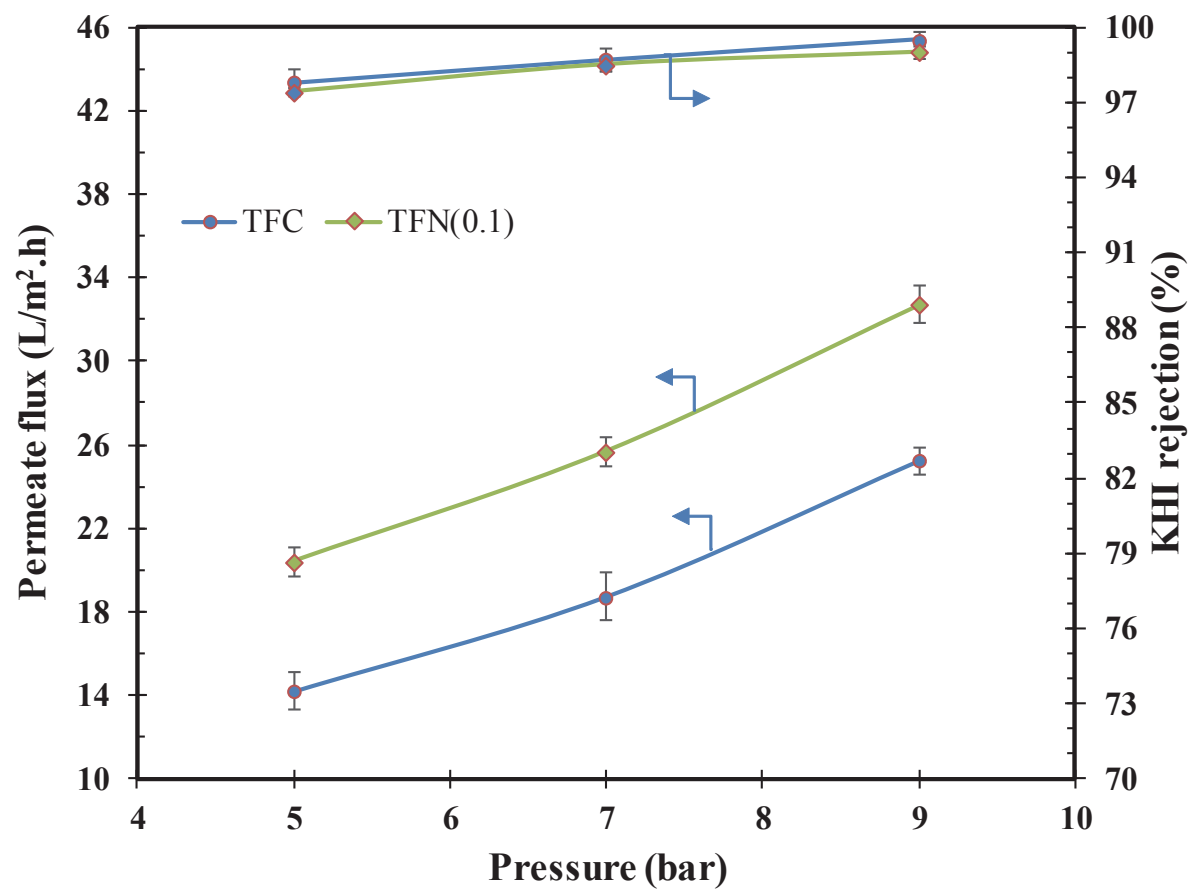


Fig. 14

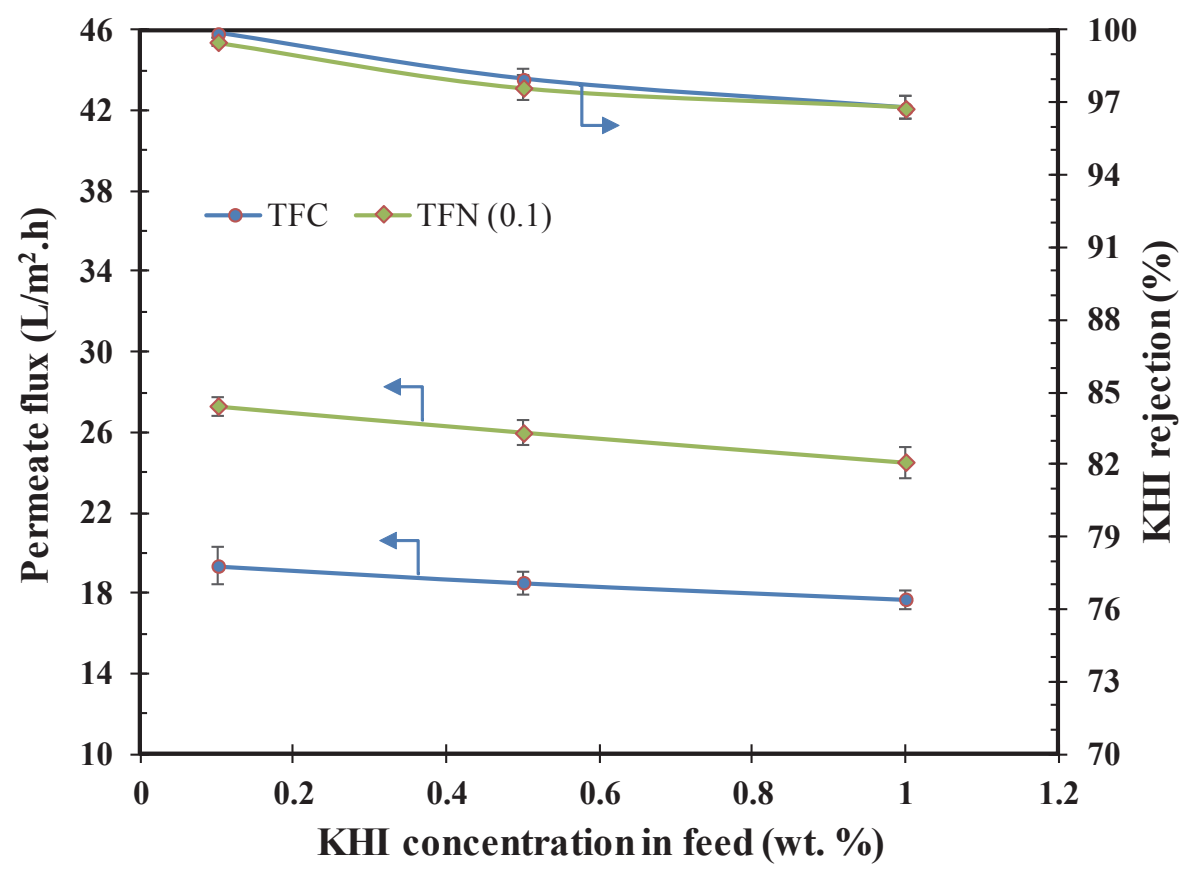


Fig. 15

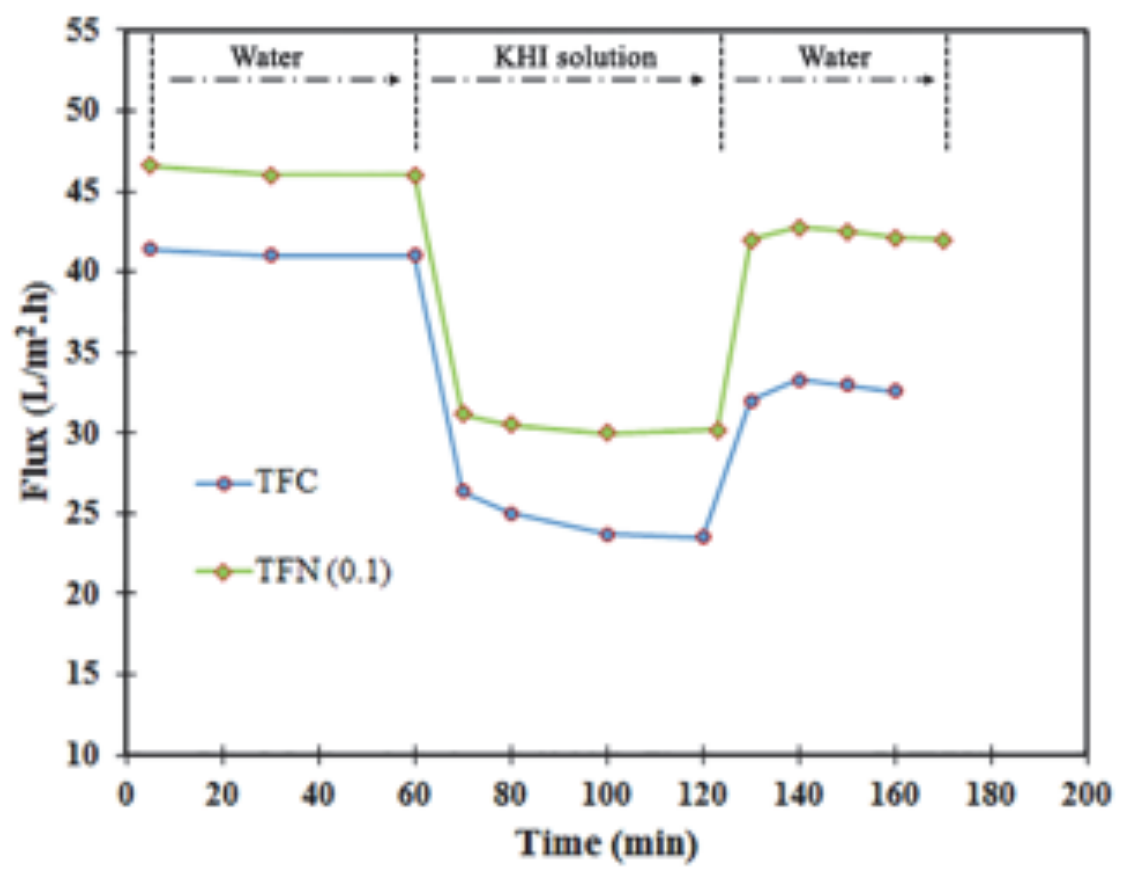




\section{Tables caption:}

Table 1. Properties of the GO used in this study.

Table 2. Composition of the prepared membranes.

Table 3. The formula for the different types of fouling.

Table 4. Effect of the GO on the properties of PPSU substrate.

Table 5. Surface roughness parameters of the support membranes via AFM analysis.

Table 6. Flux decline results and flux recovery ratio for TFC and TFN (0.1) membranes at 8 bar. 
Tables:

Table 1

\begin{tabular}{lc}
\hline Chemical structure & \\
\hline Appearance & Black powder \\
\hline Purity & $99 \%$ \\
\hline The Product COA & $\mathrm{C}=92.7 \%, \mathrm{O} \leq 7.3 \%$ \\
\hline Layers & $6-10$ Layers \\
\hline Thickness & $3.4-7 \mathrm{~nm}$ \\
\hline Diameter & $10-50 \mu \mathrm{m}$ \\
\hline Specific surface area & $100-300 \mathrm{~m}^{2} / \mathrm{g}$ \\
\hline Density & $1 \mathrm{gr} / \mathrm{cm}^{3}$ at $20{ }^{\circ} \mathrm{C}$ \\
\hline
\end{tabular}


Table 2

\begin{tabular}{lllll}
\hline Support membrane & NF membrane & GO (wt. \%) & PPSU (wt. \%) & NMP(wt. \%) \\
\hline $\mathrm{S}(0)$ & TFC & 0 & 17 & 83 \\
$\mathrm{~S}(0.05)$ & $\mathrm{TFN}(0.05)$ & 0.05 & 17 & 82.95 \\
$\mathrm{~S}(0.1)$ & $\mathrm{TFN}(0.1)$ & 0.1 & 17 & 82.9 \\
$\mathrm{~S}(0.2)$ & $\mathrm{TFN}(0.2)$ & 0.2 & 17 & 82.8 \\
$\mathrm{~S}(0.4)$ & $\mathrm{TFN}(0.4)$ & 0.4 & 17 & 82.6 \\
\hline
\end{tabular}




\section{Table 3}

\begin{tabular}{ccc}
\hline Type of fouling & Equation & Equation No. \\
\hline Reversible fouling & $(5)$ \\
Irreversible fouling & $(6)$ \\
Total fouling & (7) \\
\hline
\end{tabular}


Table 4

\begin{tabular}{lcccc}
\hline Membrane & $\boldsymbol{\varepsilon}(\%)$ & $\mathbf{r}_{\mathbf{m}}(\mathbf{n m})$ & PWF (L/m $\mathbf{2} \cdot \mathbf{h})$ & $\begin{array}{c}\text { Contact angle } \\
\left.\mathbf{(}^{\circ}\right)\end{array}$ \\
\hline $\mathrm{S}(0)$ & $56 \pm 2$ & $26.5 \pm 0.5$ & $177 \pm 2$ & $67 \pm 3$ \\
$\mathrm{~S}(0.05)$ & $57 \pm 2.1$ & $26.5 \pm 0.5$ & $180.5 \pm 2.2$ & $60 \pm 1$ \\
$\mathrm{~S}(0.1)$ & $60 \pm 0.7$ & $27.2 \pm 0.2$ & $211.5 \pm 2.5$ & $56 \pm 1$ \\
$\mathrm{~S}(0.2)$ & $59 \pm 1.4$ & $27.6 \pm 0.5$ & $205 \pm 4.2$ & $46 \pm 1.5$ \\
$\mathrm{~S}(0.4)$ & $55 \pm 1.1$ & $27 \pm 0.3$ & $174 \pm 2.9$ & $48 \pm 2.5$ \\
\hline
\end{tabular}


Table 5

\begin{tabular}{lcc}
\hline Membrane & $\mathbf{R}_{\mathbf{a}}(\mathbf{n m})$ & $\mathbf{R}_{\mathbf{q}}(\mathbf{n m})$ \\
\hline $\mathrm{S}(0)$ & 1.87 & 2.52 \\
$\mathrm{~S}(0.05)$ & 1.62 & 2.75 \\
$\mathrm{~S}(0.1)$ & 1.6 & 2.46 \\
$\mathrm{~S}(0.2)$ & 2.25 & 2.1 \\
$\mathrm{~S}(0.4)$ & 2.43 & 3.19 \\
\hline
\end{tabular}




\section{Table 6}

\begin{tabular}{|c|c|c|c|c|c|c|c|}
\hline \multirow[b]{2}{*}{ Membrane } & \multicolumn{3}{|c|}{ Flux (L/m².h) } & \multicolumn{3}{|c|}{ Flux decline (\%) } & \multirow[b]{2}{*}{ FRR (\%) } \\
\hline & $\mathrm{J}_{0}$ & $\mathrm{~J}_{1}$ & $\mathrm{~J}_{2}$ & $\begin{array}{l}\text { Caused by } \\
\text { reversible fouling }\end{array}$ & $\begin{array}{l}\text { Caused by } \\
\text { irreversible } \\
\text { fouling }\end{array}$ & Total & \\
\hline $\mathrm{TFC}$ & 41.4 & 23.5 & 32.6 & 22.0 & 21.3 & 43.2 & 78.7 \\
\hline TFN(0.1) & 46.6 & 30.2 & 42 & 25.3 & 9.9 & 35.2 & 90.1 \\
\hline
\end{tabular}


Graphical abstract:

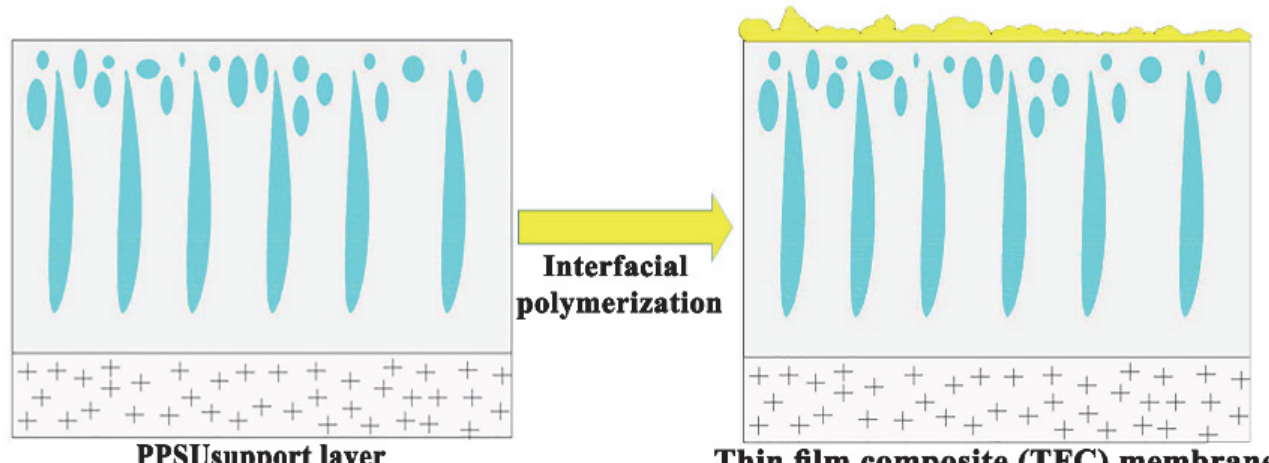

PPSUsupport layer

Thin film composite (TFC) membrane

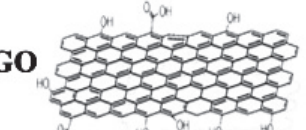

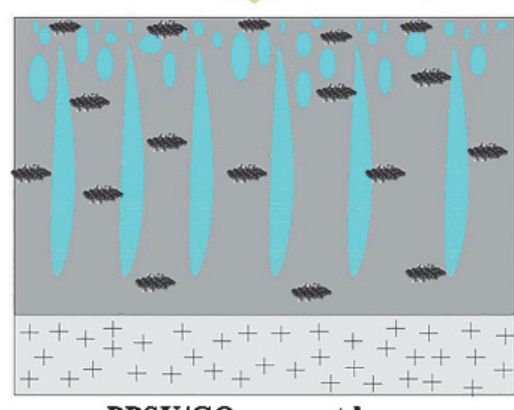

PPSU/GO support layer

* Increased Surface porosity

* Increased pore size

* Increased hydrophilicity

* Improved permeability

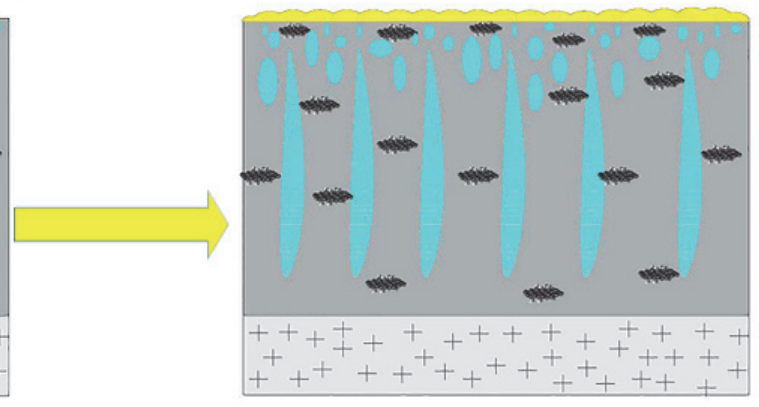

Thin film nanocomposite (TFN) membrane

* Decreased thickness of selective layer

* Decreased surface roughness

* Imroved Permeability

* Improved antifouling properties 